\title{
Analytical Dimensional Reduction of a Fuel Optimal Powered Descent Subproblem
}

\author{
Jeremy R. Rea * and Robert H. Bishop ${ }^{\dagger}$
}

\begin{abstract}
Current renewed interest in exploration of the moon, Mars, and other planetary objects is driving technology development in many fields of space system design. In particular, there is a desire to land both robotic and human missions on the moon and elsewhere. The landing guidance system must be able to deliver the vehicle to a desired soft landing while meeting several constraints necessary for the safety of the vehicle. Due to performance limitations of current launch vehicles, it is desired to minimize the amount of fuel used. In addition, the landing site may change in real-time in order to avoid previously undetected hazards which become apparent during the landing maneuver.

This complicated maneuver can be broken into simpler subproblems that bound the full problem. One such subproblem is to find a minimum-fuel landing solution that meets constraints on the initial state, final state, and bounded thrust acceleration magnitude. With the assumptions of constant gravity and negligible atmosphere, the form of the optimal steering law is known, and the equations of motion can be integrated analytically, resulting in a system of five equations in five unknowns. It is shown that this system of equations can be reduced analytically to two equations in two unknowns. With an additional assumption of constant thrust acceleration magnitude, this system can be reduced further to one equation in one unknown. It is shown that these unknowns can be bounded analytically. An algorithm is developed to quickly and reliably solve the resulting one-dimensional bounded search, and it is used as a real-time guidance applied to a lunar landing test case.
\end{abstract}

\section{Introduction}

Today is an exciting time for the exploration of the moon, Mars, and other planetary objects. Renewed interest in lunar exploration is driving new technology development for both human and robotic missions. NASA has begun a new era of space exploration with the Constellation Program, with a goal of returning to the moon to build a sustainable long term human presence. ${ }^{1}$ The Lunar X-Prize has promised to reward $\$ 30$ million to the first private team to land a rover on the moon. ${ }^{2}$ China, India, and Japan have all successfully orbited probes around the moon with hopes of someday putting down a lander. ${ }^{3,4}$ While both Russia and the United States have previously landed vehicles on the moon and Mars, new technology development is necessary to achieve greater levels of performance and safety to successfully accomplish these missions.

In the lunar landing scenario, the vehicle begins in some initial hyperbolic transfer orbit or a parking orbit around the Moon. Given the initial orbit and a desired landing point on the surface, a targeting algorithm determines the best Time-of-Ignition (TIG) at which to begin the deorbit. A burn is completed to put the lander in a coasting elliptical transfer orbit. At the designated time, the powered terminal descent phase begins. This phase can be broken into a braking phase and an approach phase. ${ }^{5}$ During the braking phase, much of the velocity relative to the planet is reduced. During the approach phase, a pitch up maneuver places the vehicle in a vertical attitude above the landing site. The vehicle then descends vertically to the surface. During any part of the braking or approach phases, hazard avoidance may necessitate a target redesignation.

\footnotetext{
*Aerospace Engineer, Flight Mechanics and Trajectory Design Branch, NASA Johnson Space Center, AIAA Member.

${ }^{\dagger}$ Professor, Aerospace Engineering, The University of Texas at Austin, AIAA Member.
} 


\section{Current and Suggested Guidance Methods for Powered Landing}

\section{II.A. Apollo Lunar Descent Guidance}

The heart of the Apollo lunar descent guidance is the assumption that the thrust acceleration is a quadratic function of time. This defines a two-point boundary value problem with five degrees of freedom which allows for the specification of the initial position, initial velocity, final position, final velocity, and final acceleration. These equations can be solved for the coefficients as a function of the known initial position and velocity, the desired final position, velocity, and acceleration, and the time-to-go $\left(t_{g o}\right)$. The result is a simple guidance algorithm that produces feasible trajectories and which could run on the Apollo flight computer. In terms of fuel used, it has been shown that the Apollo guidance performs well when compared to a fuel optimal trajectory when only small target redesignations are necessary. However, when large target redesignations are needed, the Apollo guidance uses much more fuel than a fuel optimal trajectory. ${ }^{6,7}$ Also, care must be taken to define a proper value of $t_{g o}$ in order that the trajectory meet the desired characteristics. More details of the Apollo lunar descent guidance can be found in references 8, 9, and 10.

\section{II.B. Powered Explicit Guidance (PEG)}

The Powered Explicit Guidance (PEG) algorithm is used by the Space Shuttle for near fuel-optimal exoatmospheric powered maneuvers. It was derived for powered ascent trajectories which end in an orbital injection. As such, there is no constraint on the downrange position. Beginning with the optimal control law for powered flight over a flat Earth in a uniform gravitational field, it is assumed that the unit thrust direction is mainly in the downrange direction (an assumption that holds for most Space Shuttle maneuvers). A further small angle assumption is made such that the unit thrust direction vector is approximated to be a linear function of time. With these assumptions, the equations of motion can be integrated analytically to produce a system of nine equations in nine unknowns. All variables can be computed given a value of the velocity-tobe-gained vector $\left(\vec{V}_{g o}\right)$. A predictor-corrector algorithm is used to find the solution, given an initial guess for $\vec{V}_{g o}$ and the desired final velocity and position vectors (except the downrange component of position). ${ }^{11-13}$ Delporte and Sauvient reported an extension of the explicit guidance concept with constraints on the guidance law, such as altitude at first stage engine cut-off, thermal flux at first stage separation, and the landing footprint of the first stage. ${ }^{14}$ Fill expanded the PEG equations to include a constraint on downrange. ${ }^{12}$ This allows for a solution with a fully constrained final position and velocity, such as that of a powered terminal descent. Unfortunately, this expanded capability was not implemented in the PEG flight software.

As can be seen, there are many approximations made which reduce the optimality of PEG. There is also no way to completely constrain the final position with the original implementation; however, Fill's improvements should allow for this. In addition, while PEG is able to combine multiple thrust arcs, such as a constant thrust arc followed by a constant acceleration arc, there is no computation of optimal switching times for the throttle command between maximum and minimum thrust levels. In fact, PEG was derived assuming that the thrust profile is a known function of time.

\section{II.C. Other Methods}

These methods have been suggested but have never flown on an actual space vehicle.

\section{II.C.1. Analytical Near Fuel-Optimal}

Since the fuel-optimal problem is difficult to solve, it has been proposed to solve related problems that are near fuel-optimal. D'Souza developed a guidance algorithm for powered vacuum flight in a uniform gravitational field by minimizing a weighted function of the time-of-flight and the square of the acceleration magnitude. ${ }^{15}$ Two similar ideas are proposed by Najson and Mease: 1) minimize the integral of the sum of the absolute values of the thrust acceleration components, and 2) minimize the square of the thrust acceleration magnitude. ${ }^{16}$ Application of optimal control theory results in an analytical solution in terms of the initial position/velocity state, desired final position/velocity state, and time-of-flight. D'Souza analytically calculated the optimal time-of-flight as a function of the state, while Najson and Mease used a numerical search to find the time-of-flight with the smallest fuel expenditure. For an engine with a constant specific impulse $\left(I_{s p}\right.$, Ross has shown that a fuel optimal solution is found by minimizing the integral of the magnitude of the thrust acceleration. ${ }^{17}$ Thus, while these methods are fuel efficient, they are not fuel optimal. 
Ueno and Yamaguchi derived an analytical guidance law by making several approximations. ${ }^{18}$ Starting with the problem of fuel-optimal planar flight in a uniform gravitational field with a constant thrust acceleration, a small angle assumption is made that assumes the flight is mostly horizontal with the thrust being directed nearly opposite the velocity vector. Constraints are placed on the final velocity vector and the final altitude, and the final crossrange and downrange are free. Optimal control theory then leads to a polynomial guidance law where the downrange and crossrange velocities are linear functions of time, the altitude rate is a quadratic function of time, and the altitude is a cubic function of time. This guidance algorithm is shown to work well for the defined problem. However, it does not allow for designation of a specific landing site, except by proper selection of the deorbit state. This fact and the assumption of near horizontal flight implies that the proposed algorithm will not work for hazard avoidance maneuvers. The approximations also imply that the problem is not truly fuel optimal.

Uchiyama used a barrier function method to transform the fuel optimization problem with bounded thrust magnitude into an unconstrained problem. ${ }^{19}$ The controls are taken as the total acceleration components along the radial and local horizontal directions, including both the thrust and gravitational acceleration terms. The magnitude of the total acceleration is then minimized. In order to find the necessary thrust acceleration commands, the gravitational vector is subtracted from the optimal total acceleration vector. Issues with this approach include the lack of a constraint on the thrust acceleration magnitude and a lack of fuel optimality.

\section{II.C.2. Analytical Gravity Turn}

McInnes and Chomel developed guidance algorithms based on the gravity turn where the thrust vector is directed opposite the velocity vector. ${ }^{20,21}$ The gravity turn has the desirable properties that the final velocity magnitude is zero and the final vehicle attitude is vertical. For planar motion and constant thrust acceleration, it is possible to analytically integrate the equations of motion for altitude, downrange, velocity magnitude, and time as a function of flight path angle. The trajectory can be shaped by breaking it into segments with different thrust acceleration magnitudes. This guidance algorithm is shown to work well, and Chomel has demonstrated that it can be used for target redesignations. However, while the gravity turn may be near fuel-optimal for descent from orbit, it is not a fuel optimal maneuver for target redesignations near the end of the terminal descent trajectory. Thus, while this method is a viable solution for an analytical terminal descent guidance, it may not be applicable to all terminal descent situations.

\section{II.C.3. Numerical Methods}

Acikmese and Ploen have suggested using a direct numerical method to solve the powered terminal descent problem. ${ }^{22}$ The problem of time-fixed, fuel-optimal flight in a uniform gravitational field is made convex under certain assumptions. This convex problem is then converted into a second-order cone programming problem (SOCP) and solved using a numerical interior point method to find a global optimum with a known upper bound on the number of iterations. An outer iteration is necessary to find the optimal time-of-flight. The proposed guidance algorithm would numerically solve the SOCP onboard the vehicle. This method has the advantage of allowing several constraints, such as bounded thrust, bounded vehicle attitude during flight, constrained final vehicle attitude, and constraints on altitude such that the vehicle does not fly subsurface. While this approach holds much potential, it has the disadvantage of using a numerical solver. Also, the outer iteration to find the best time-of-flight is undesirable since the SOCP must be solved multiple times. While a solution to the SOCP can theoretically always be found, it would be difficult to verify convergence for all possible scenarios. Cognizant of these issues, a method was proposed whereby a family of optimal trajectories would be generated pre-flight using this method, and a table look-up would be used onboard to generate near fuel-optimal trajectories for any initial state. ${ }^{23}$

\section{II.D. Areas for Improvement and Scope of Work}

A fuel-optimal guidance algorithm is needed for hazard avoidance and precision landing. It can be seen that many methods for powered terminal descent have been proposed. Only the Apollo Lunar Descent Guidance and the Shuttle Powered Explicit Guidance have been proven in flight. Most of the methods either make no use of optimal control theory, make approximations of the fuel optimal control law, or minimize performance functions that do not lead to fuel optimal solutions. Current mission designs put a high value on any design 
feature that can reduce mass, thus a fuel optimal solution is desirable. Most of the methods also do not have the ability to bound the pitch angle of the vehicle during flight. This capability may be required to ensure that the vehicle attitude allows astronauts or sensors to view the landing site through a window. Some of the methods can be used for hazard avoidance maneuvers and some can not.

It is desired to find a guidance algorithm which is fuel-optimal while meeting constraints on the final state, thrust magnitude, sensor and/or window viewing angles, and minimum approach altitude. The guidance must be able to generate commands in real-time without relying on the solution from the previous guidance cycle, as in the case of a target redesignation in mid-flight due to hazard avoidance at the original landing site. It is likely that this challenging problem has no analytical solution. However, it can be broken down into simpler subproblems which can be solved analytically. The solutions to these subproblems may be useful bounds for a numerical solution of the full problem. Towards that goal, this paper investigates the simpler subproblem of fuel optimal flight with bounded thrust acceleration magnitude from a given initial state to a desired final state; the pitch up maneuver to place the lander in the desired final vertical attitude is not considered. It will be shown that this problem can be reduced to an analytically bounded search with excellent convergence properties.

\section{Problem Definition}

The following assumptions are made:

1. Atmospheric forces can be neglected.

2. Gravitational acceleration is constant.

3. The rotation of the planetary object can be neglected.

4. The vehicle carries a perfectly expanded chemical rocket engine with which to create thrust force.

5. The nozzle exit velocity of the propellant is a known constant.

6. The thrust magnitude has known limits.

7. The thrust direction is along the roll axis of the vehicle and can be commanded in any direction instantaneously.

8. The final time is free.

The only forces acting on the vehicle are the force due to gravity and its own thrust force. The equations of motion are:

$$
\begin{aligned}
\dot{\vec{R}} & =\vec{V} \\
\dot{\vec{V}} & =a_{T} \hat{u}+\vec{g}
\end{aligned}
$$

where $\vec{R}$ is the position vector, $\vec{V}$ is the velocity vector, $\vec{g}$ is the gravitational acceleration vector, $a_{T}$ is the magnitude of the thrust acceleration, and $\hat{u}$ is the unit thrust direction vector.

It is desired to minimize the propellant used during the maneuver. It can be shown that minimizing the amount of propellant burned is equivalent to minimizing the integral of the magnitude of the thrust acceleration. ${ }^{24}$ Note that no assumption of constant thrust or constant mass is made.

$$
\left\{\min \quad J=m_{o}-m_{f}\right\} \quad \rightleftarrows\left\{\min \quad J=\int_{t_{o}}^{t_{f}} a_{T} d t\right\}
$$

where $m_{o}$ is the initial vehicle mass and $m_{f}$ is the final vehicle mass.

There is a maximum rate at which propellant mass can be expelled from the chemical rocket engine. The absolute minimum rate would be zero. However, there may be operating conditions such that the minimum rate is nonzero. These limits on propellant mass flow rate translate directly into limits on thrust magnitude. Note that it is not assumed that the thrust magnitude limits are constant.

$$
0 \leq T_{\min }(t) \leq T \leq T_{\max }(t)
$$


Dividing equation 3 by the mass and substituting $a_{T}=\frac{T}{m}$ yields

$$
0 \leq \frac{T_{\min }(t)}{m(t)} \leq a_{T} \leq \frac{T_{\max }(t)}{m(t)}
$$

The thrust acceleration magnitude limits are rewritten as:

$$
0 \leq a_{T_{\min }}(t) \leq a_{T} \leq a_{T_{\max }}(t)
$$

Constraints are placed on the final state such that:

$$
\psi=\left\{\begin{array}{l}
\sigma_{\mathrm{R}_{\mathrm{x}}}\left(R_{x_{f}}-R_{x_{f_{s}}}\right)=0 \\
\sigma_{\mathrm{R}_{\mathrm{y}}}\left(R_{y_{f}}-R_{y_{f_{s}}}\right)=0 \\
\sigma_{\mathrm{R}_{\mathrm{z}}}\left(R_{z_{f}}-R_{z_{f_{s}}}\right)=0 \\
\sigma_{V_{\mathrm{x}}}\left(V_{x_{f}}-V_{x_{f_{s}}}\right)=0 \\
\sigma_{V_{\mathrm{y}}}\left(V_{y_{f}}-V_{y_{f_{s}}}\right)=0 \\
\sigma_{V_{\mathrm{z}}}\left(V_{z_{f}}-V_{z_{f_{s}}}\right)=0
\end{array}\right.
$$

where

$$
\begin{aligned}
\sigma_{i} & =\text { on/off switch for each constraint }(=0-\text { OR- } 1) \\
\vec{R}_{f} & =\text { final position vector } \\
\vec{V}_{f} & =\text { final velocity vector } \\
\vec{R}_{f_{s}} & =\text { specified final position vector } \\
\vec{V}_{f_{s}} & =\text { specified final velocity vector }
\end{aligned}
$$

The $\sigma_{i}$ are added to allow easy comparison of solutions with different final constraints.

\section{Application of Optimal Control Theory}

The problem can be solved with the well known techniques of optimal control theory, and it is a very well solved problem. As such, the results will be quoted directly here. Details of the solution can be found in references 24-33, as well as others. In this research, the terminology and methodology from reference 34 are used. An assumption of familiarity with these methods is assumed. The Hamiltonian is given by:

$$
H=a_{T}+\vec{\lambda}_{R} \cdot \vec{V}+\vec{\lambda}_{V} \cdot\left[a_{T} \hat{u}+\vec{g}\right]
$$

where $\vec{\lambda}_{R}$ and $\vec{\lambda}_{V}$ are the costate Lagrange multipliers associated with position and velocity, respectively. Define the following variables:

$$
\begin{gathered}
\vec{C}_{R}=\left[\begin{array}{l}
C_{R_{x}} \\
C_{R_{y}} \\
C_{R_{z}}
\end{array}\right]=\left[\begin{array}{l}
\nu_{R_{x}} \sigma_{R_{x}} \\
\nu_{R_{y}} \sigma_{R_{y}} \\
\nu_{R_{z}} \sigma_{R_{z}}
\end{array}\right] \\
\vec{C}_{V}=\left[\begin{array}{l}
C_{V_{x}} \\
C_{V_{y}} \\
C_{V_{z}}
\end{array}\right]=\left[\begin{array}{l}
\nu_{V_{x}} \sigma_{V_{x}} \\
\nu_{V_{y}} \sigma_{V_{y}} \\
\nu_{V_{z}} \sigma_{V_{z}}
\end{array}\right]
\end{gathered}
$$

The Lagrange multipliers are continuous functions and can be written as: ${ }^{24}$

$$
\begin{aligned}
& \vec{\lambda}_{R}=\vec{C}_{R} \\
& \vec{\lambda}_{V}=\vec{C}_{V}+\vec{C}_{R} \tau
\end{aligned}
$$

Let the time-to-go from the current time be defined as

$$
\tau=\left(t_{f}-t\right)
$$

The total time-of-flight is defined as

$$
\tau_{o}=\left(t_{f}-t_{o}\right)
$$


The length of $\vec{\lambda}_{V}$ is given by

$$
\lambda_{V}=\left|\vec{\lambda}_{V}\right|=\sqrt{D+2 E \tau+F \tau^{2}}
$$

where

$$
\begin{aligned}
D & =\vec{C}_{V} \cdot \vec{C}_{V} \\
E & =\vec{C}_{R} \cdot \vec{C}_{V} \\
F & =\vec{C}_{R} \cdot \vec{C}_{R}
\end{aligned}
$$

\section{IV.A. Bilinear Tangent Steering}

Lawden was the first to have derived the linear tangent steering law for powered vacuum flight in a uniform gravitational field. ${ }^{25,26}$ Miele showed that the most general optimal control law for planar powered vacuum flight in a uniform gravitational field is the bilinear tangent steering law. ${ }^{27}$ In vector notation, the optimal

unit thrust vector in a uniform gravitational field is opposite the direction of the $\vec{\lambda}_{V}$ Lagrange vector, or "primer" vector: ${ }^{24}$

$$
\hat{u}=-\frac{\vec{\lambda}_{V}}{\left|\vec{\lambda}_{V}\right|}=-\frac{\left(\vec{C}_{V}+\vec{C}_{R} \tau\right)}{\sqrt{D+2 E \tau+F \tau^{2}}}
$$

If the motion is restricted to the XZ-plane and the angle of the unit thrust vector measured from the local horizontal, $\theta$, is found, then equation 15 reduces to the more familiar bilinear tanget form:

$$
\tan \theta=\frac{C_{V_{Z}}+C_{R_{Z}} \tau}{C_{V_{X}}+C_{R_{X}} \tau}
$$

Note that the structure of the bilinear tangent steering law is independent of the thrust function; the form of the bilinear tangent steering law holds for any bounded thrust profile. It is also independent of the optimization function. However, this steering law can reduce to various forms, such as linear tangent or constant thrust direction, depending on the optimization function, initial constraints, and final constraints. ${ }^{27}$ For example, when the downrange component of position is unconstrained, the bilinear tangent law reduces to a linear tangent law. ${ }^{28,29}$

The derivation of the classic bilinear tangent steering law uses the assumption of a uniform gravitational field. The gravitational acceleration can be reasonably assumed constant when the change in radial distance from the center of the planetary object during the maneuver is small. Yang found that when the bilinear tangent steering law is used in an inverse square gravitational field, it "is an excellent approximation to the true optimal control for powered flight arcs up to at least 15 degrees" of central arc. ${ }^{35}$ In addition, it is possible that this approximation may hold reasonably well for trajectories with larger central arcs.

\section{IV.B. "Bang-Bang" Thrust Profile}

Leitmann showed that for a bounded thrust problem, the optimal thrust profile consists of up to three maximum or minimum thrust arcs. The structure of the thrust profile is such that the possible arcs include: max, max-min, max-min-max, min-max, or min. He showed that the use of intermediate thrust arcs is not optimal in most situations. In addition, he showed that the Hamiltonian, $H$, and costates, $\vec{\lambda}_{R}$ and $\vec{\lambda}_{V}$, are continuous across the thrust switches. ${ }^{30,31}$ This means that the bilinear tangent steering law is a continuous function even when the thrust magnitude switches instantaneously. A solution to the special case of pure vertical landing was found by Meditch. He showed that the optimal thrust profile would be either $\max$ or min-max. ${ }^{36}$ These thrust profiles are called "bang-bang" because the thrust magnitude "bangs" instantaneously between its maximum and minimum possible values. Note that modern rocket engines can be throttled very quickly. Topcu, Casoliva, and Mease noted that the engines for the Mars Smart Lander, which is to be launched in 2011, can be "throttled between minimum and maximum thrust in 30-40 ms, and so the thrust magnitude dynamics are very fast compared with the translational and mass dynamics" ${ }^{32}$ This means that guidance commands that call for instantaneous thrust switches between maximum and minimum limits will not pose a problem for modern planetary landers. 
The thrust acceleration magnitude switches between its upper and lower limits according to the value of the thrust acceleration magnitude switching structure (SS). ${ }^{24}$

$$
S S=-\left(\vec{\lambda}_{V} \cdot \hat{u}\right)=\left|\vec{\lambda}_{V}\right|=\sqrt{D+2 E \tau+F \tau^{2}}
$$

Recall that the Lagrange multipliers $\vec{\lambda}_{V}$ are continuous. Therefore, the switching structure is continuous. The thrust acceleration magnitude switching condition is given by

$$
\begin{array}{lll}
S S \leq 1 & \text { when } & a_{T}=a_{T_{\min }} \\
S S \geq 1 & \text { when } & a_{T}=a_{T_{\max }} \\
S S=1 & \text { when } & a_{T_{\min }}<a_{T}<a_{T_{\max }}
\end{array}
$$

One question to ask is when an optimal, off-boundary acceleration subarc can occur. It is known that this can occur only when SS =1. By examining the form of the SS, it can be seen that off-boundary acceleration subarcs will generally occur only instantaneously. However, there is a special case when the thrust acceleration can be continuously off-boundary for an entire trajectory. Examination of equation 17 shows that a continuous off-boundary acceleration subarc can occur if

$$
\begin{aligned}
& D=\vec{C}_{V} \cdot \vec{C}_{V}=1 \\
& E=\vec{C}_{R} \cdot \vec{C}_{V}=0 \\
& F=\vec{C}_{R} \cdot \vec{C}_{R}=0
\end{aligned}
$$

This implies that a continuous off-boundary acceleration subarc can occur when $\vec{C}_{R}=\vec{\lambda}_{R}=0$. Thus, for an off-acceleration boundary subarc:

$$
\vec{\lambda}_{V}=\vec{C}_{V}=\text { constant vector }
$$

Recall that the Lagrange multiplier $\vec{\lambda}_{V}$ is continuous on an optimal trajectory. This implies that it has the same constant value for the entire trajectory. Thus, for an off-acceleration subarc to occur, the entire optimal trajectory must be off the acceleration boundaries. This means that the unit thrust direction vector will have the same constant value for the entire optimal trajectory. Thus, for an off-acceleration boundary subarc, we have

$$
\hat{u}=\text { constant unit vector }
$$

Recall that when $\mathrm{SS}=1$, this means that $\left|\vec{\lambda}_{V}\right|=\sqrt{D+2 E \tau+F \tau^{2}}=1$. For the special case when the thrust acceleration is off-boundary for an entire subarc, $\vec{C}_{R}=\vec{\lambda}_{R}=0$. Also, $\vec{\lambda}_{V}=$ constant. Thus, the Hamiltonian in equation 23 becomes

$$
H=\vec{\lambda}_{V} \cdot \vec{g}=\vec{C}_{V} \cdot \vec{g}=0
$$

This implies that $\vec{\lambda}_{V}$ must be perpendicular to $\vec{g}$. This implies that the unit thrust direction is in the horizontal plane.

From equation $8, \vec{C}_{R}$ is zero when $\vec{\sigma}_{R}$ is zero. Recall from equation 6 that the $\vec{\sigma}_{R}$ are binary variables that act as on/off switches for the final position constraints. Thus, there are two cases when an off-boundary thrust acceleration solution is possible. The first case is when no final position is specified. The second case is when the desired final position is achieved by chance without a specific constraint on the final position. In this case, the thrust directions will only allow for a very limited set of trajectories. Thus, while this case should not be ignored, it will almost never appear in most real-world problems if the full final state is specified. Thus, most problems will have a thrust acceleration on one of its boundaries with instantaneous switches between the boundaries. However, it should be noted that although it is only possible to have an off-boundary acceleration solution when there is no final position constraint, it is also possible to have an on-boundary acceleration solution when there is no final position constraint. However, an on-boundary acceleration solution with no final position constraint will not have the condition of equation 22 . This is discussed further in Section XI. 


\section{IV.C. Integrals of the Problem}

Certain first integrals of the optimization problem are known.

\section{- Hamiltonian}

For a time independent gravitational field, the Hamiltonian is constant. In addition, if the final time of the problem is free, the Hamiltonian has a constant value of zero. ${ }^{33}$ Substiting for the optimal unit thrust direction into equation 7 , the first integral of the problem is then

$$
\begin{aligned}
& H=a_{T}\left[1-\left|\vec{\lambda}_{V}\right|\right]+\vec{\lambda}_{R} \cdot \vec{V}+\vec{\lambda}_{V} \cdot \vec{g}=0 \\
& H=a_{T}\left[1-\sqrt{D+2 E \tau+F \tau^{2}}\right]+\left(\vec{C}_{R} \cdot \vec{V}\right)+\left(\vec{C}_{V}+\vec{C}_{R} \tau\right) \cdot \vec{g}=0
\end{aligned}
$$

\section{- Rotational Symmetry}

The optimal powered flight problem has an axis of symmetry. Problems in an inverse gravitational field have a spherical symmetry. This leads to the following relationship:

$$
\vec{R} \times \vec{\lambda}_{R}+\vec{V} \times \vec{\lambda}_{V}=\text { Constant (Inverse gravitational field) }
$$

A derivation of this relationship can be found in reference 33 (pp. 85-86). For problems with a uniform gravitational field, the axis of symmetry is about the gravitational vector. Thus, the component of the vector expressed in equation 24 that is parallel to the gravitational vector is constant. ${ }^{32,33}$

$$
\left[\vec{R} \times \vec{\lambda}_{R}+\vec{V} \times \vec{\lambda}_{V}\right] \cdot \vec{g}=\text { Constant (Uniform gravitational field) }
$$

\section{Analytical Integration of the Equations of Motion}

It is possible to analytically integrate the equations of motion with the bilinear tangent steering law defined in equation 15 using standard integral tables. Yang integrated the equations with constant thrust magnitude. ${ }^{37}$ Fill integrated them assuming that the thrust acceleration profile is a quadratic function of time. ${ }^{12}$ Delporte and Sauvient integrated the equations for a constant thrust and the assumption that the two vectors defining the bilinear tangent law are perpendicular $\left(\vec{C}_{R} \cdot \vec{C}_{V}=0\right) .{ }^{14}$ Feeley integrated both the linear tangent guidance law constrained to a plane and the three dimensional bilinear tangent guidance law, both with constant thrust magnitude. ${ }^{38,39}$ Leung, Calise, and Hull integrated the equations of motion constrained to a plane with the bilinear tangent law and constant thrust magnitude. ${ }^{40,41}$

For all these integrations, the result is a system of nonlinear equations for the final state as a function of the vectors defining the bilinear tangent law and the time-of-flight. This system of equations is difficult to solve, and in all the references where analytical integration was completed, the resulting system of nonlinear equations is solved using numerical methods. Indeed, Yang himself expressed that once the equations of motion are integrated analytically, "the determination of the constants involves the solving of 15 coupled nonlinear simultaneous equations. It is impractical to do it analytically. With the assistance of computers, a numerical scheme is much more appealing". ${ }^{37}$ However, it will be shown that analytical reduction of the problem is possible and yields useful results.

It is convenient to integrate the equations of motion in terms of the time-to-go, $\tau$, defined in equation 11. This change of variable is equivalent to integrating the equations of motion backwards from the final time. Consider the integration of the trajectory from the final time of flight to the initial time of flight. At $t=t_{f}, \tau_{f}=0, \vec{V}=\vec{V}_{f}$ and $\vec{R}=\vec{R}_{f}$. At $t=t_{o}, \tau=\tau_{o}=\left(t_{f}-t_{o}\right), \vec{V}=\vec{V}_{o}$ and $\vec{R}=\vec{R}_{o}$. After completing the integrations and rearranging the equations, the following vectors can be defined:

$$
\begin{aligned}
\tilde{V} & :=\left(\vec{V}_{f}-\vec{V}_{o}\right)-\vec{g} \tau_{o}=\left[\int_{0}^{\tau_{o}} a_{T} \hat{u} d \tau\right] \\
\tilde{R} & :=\left(\vec{R}_{f}-\vec{R}_{o}\right)-\vec{V}_{o} \tau_{o}-\frac{1}{2} \vec{g} \tau_{o}^{2}=\left[\int_{0}^{\tau_{o}} \int_{0}^{\tau_{o}} a_{T} \hat{u} d \tau d \tau\right]
\end{aligned}
$$

It is known that the optimal thrust acceleration magnitude will have up to three phases, with the thrust acceleration magnitude equal to either its minimum or maximum value. Only in certain very rare situations, 
it is possible for $a_{T}$ to have a continuous, time-varying, off-boundary solution with a constant unit thrust direction. These cases will not be considered further. At this point, it will be assumed that the thrust acceleration limits are constant (i.e. $a_{T_{\min }}=$ constant, $a_{T_{\max }}=$ constant). This means that $a_{T}$ will have a constant value on each phase. It can be shown that the same general solution exists for optimal trajectories with one, two, or three thrust acceleration subarcs, that is

$$
\begin{aligned}
\tilde{V} & =\left(\frac{P_{i}}{F}-\frac{E G_{i}}{\sqrt{F^{3}}}\right) \vec{C}_{R}+\left(\frac{G_{i}}{\sqrt{F}}\right) \vec{C}_{V} \\
\tilde{R} & =\frac{1}{2}\left[\left(3 \frac{E^{2}}{\sqrt{F^{5}}}-\frac{D}{\sqrt{F^{3}}}\right) G_{i}-2 \frac{K_{i}}{F}-3 \frac{E P_{i}}{F^{2}}\right] \vec{C}_{R}+\left(\frac{P_{i}}{F}-\frac{E G_{i}}{\sqrt{F^{3}}}\right) \vec{C}_{V}
\end{aligned}
$$

These equations represent the general integrated equations of motion for optimal solutions. They apply regardless of how many thrust acceleration subarcs exist (one, two, or three). $P_{i}, G_{i}$, and $K_{i}$ are integration constants where the subscript $i$ refers to the number of subarcs. Details of the integration can be found in reference 24 .

\section{Thrust Vector Plane}

Lawden observed that the optimal thrust vector from equation 15 lies in an inertial plane defined by the two constant vectors $\vec{C}_{R}$ and $\vec{C}_{V}^{26}$ (pp. 70-71). It never leaves this plane. From equation 27, it can be seen that the two vectors $\tilde{V}$ and $\tilde{R}$ can be written as linear combinations of $\vec{C}_{R}$ and $\vec{C}_{V}$. Thus, all four vectors are coplanar. Define this plane as the Thrust Vector Plane, or simply the Thrust Plane (TP). A coordinate system can be set in this plane with the Z-axis perpendicular to the thrust plane. Marec observed that the vectors $\vec{C}_{R}$ and $\vec{C}_{V}$ can be written in terms of a magnitude and an angle ${ }^{29}$ (p. 72). A seemingly new observation, but one that is obvious, is that the thrust plane is defined entirely by $\tilde{V}$ and $\tilde{R}$, which are functions of the initial state, final state, gravity, and time-of-flight. This fact can be used to greatly simplify the problem.

Define a cartesian coordinate system such that the Z-axis points up along the vertical direction with the origin at an altitude of zero. The $\mathrm{X}$-axis and $\mathrm{Y}$-axis form the horizontal plane. This coordinate system will be referred to as the inertial frame and will be denoted with a subscript of "I". A subscript of "TP" will be used to denote vectors on the Thrust Plane. Define the Thrust Plane X-axis along the direction of $\tilde{V}_{I}$. Define the Thrust Plane Z-axis as a unit vector perpendicular to the plane. The Thrust Plane Y-axis completes the right-handed coordinate system. The axes of the Thrust Plane Coordinate System are then defined in terms of inertial vectors as

$$
\begin{aligned}
\hat{x}_{T P_{I}} & =\frac{\tilde{V}_{I}}{\left|\tilde{V}_{I}\right|} \\
\hat{y}_{T P_{I}} & =\hat{z}_{T P_{I}} \times \hat{x}_{T P_{I}} \\
\hat{z}_{T P_{I}} & =\frac{\tilde{V}_{I} \times \tilde{R}_{I}}{\left|\tilde{V}_{I} \times \tilde{R}_{I}\right|}
\end{aligned}
$$

Define the angle $\phi$ as the angle of the vector of interest off of the Thrust Plane. Define the angle $\theta$ as the angle in the plane between the vector of interest and the $\tilde{V}$ vector (i.e. Thrust Plane X-axis), measured positive about the Thrust Plane normal. The vectors $\vec{R}_{o}, \vec{V}_{o}, \vec{R}_{f}, \vec{V}_{f}$, and $\vec{g}$ become:

$$
\begin{aligned}
\vec{R}_{o} & =R_{o}\left(\cos \phi_{R_{o}} \cos \theta_{R_{o}} \hat{i}_{T P}+\cos \phi_{R_{o}} \sin \theta_{R_{o}} \hat{j}_{T P}+\sin \phi_{R_{o}} \hat{k}_{T P}\right) \\
\vec{V}_{o} & =V_{o}\left(\cos \phi_{V_{o}} \cos \theta_{V_{o}} \hat{i}_{T P}+\cos \phi_{V_{o}} \sin \theta_{V_{o}} \hat{j}_{T P}+\sin \phi_{V_{o}} \hat{k}_{T P}\right) \\
\vec{R}_{f} & =R_{f}\left(\cos \phi_{R_{f}} \cos \theta_{R_{f}} \hat{i}_{T P}+\cos \phi_{R_{f}} \sin \theta_{R_{f}} \hat{j}_{T P}+\sin \phi_{R_{f}} \hat{k}_{T P}\right) \\
\vec{V}_{f} & =V_{f}\left(\cos \phi_{V_{f}} \cos \theta_{V_{f}} \hat{i}_{T P}+\cos \phi_{V_{f}} \sin \theta_{V_{f}} \hat{j}_{T P}+\sin \phi_{V_{f}} \hat{k}_{T P}\right) \\
\vec{g} & =g\left(\cos \phi_{g} \cos \theta_{g} \hat{i}_{T P}+\cos \phi_{g} \sin \theta_{g} \hat{j}_{T P}+\sin \phi_{g} \hat{k}_{T P}\right)
\end{aligned}
$$


Note that the vectors $\vec{C}_{R}, \vec{C}_{V}, \tilde{R}$, and $\tilde{V}$ lie on the Thrust Plane. Thus $\phi=0$ for each vector, and they have no Z-component. So, on the Thrust Plane we have

$$
\begin{aligned}
\tilde{V}_{T P} & =\tilde{V}_{\text {mag }}\left(\cos \theta_{\tilde{V}} \hat{i}_{T P}+\sin \theta_{\tilde{V}} \hat{j}_{T P}\right) \\
\tilde{R}_{T P} & =\tilde{R}_{\text {mag }}\left(\cos \theta_{\tilde{R}} \hat{i}_{T P}+\sin \theta_{\tilde{R}} \hat{j}_{T P}\right) \\
\vec{C}_{V_{T P}} & =C_{V}\left(\cos \theta_{C_{V}} \hat{i}_{T P}+\sin \theta_{C_{V}} \hat{j}_{T P}\right) \\
\vec{C}_{R_{T P}} & =C_{R}\left(\cos \theta_{C_{R}} \hat{i}_{T P}+\sin \theta_{C_{R}} \hat{j}_{T P}\right)
\end{aligned}
$$

where

$$
\begin{aligned}
\tilde{V}_{\text {mag }} & =\text { Magnitude of the } \tilde{V} \text { vector } \\
\tilde{R}_{\text {mag }} & =\text { Magnitude of the } \tilde{R} \text { vector } \\
C_{V} & =\text { Magnitude of the } \vec{C}_{V} \text { vector } \\
C_{R} & =\text { Magnitude of the } \vec{C}_{R} \text { vector }
\end{aligned}
$$

Note that $\theta_{\tilde{V}}=0$ by definition, because the X-Thrust Plane axis is in the direction of $\tilde{V}$. However, for purposes of a more general derivation, this fact will be ignored for now. Define

$$
\Phi:=\left(\cos \theta_{C_{R}} \cos \theta_{C_{V}}+\sin \theta_{C_{R}} \sin \theta_{C_{V}}\right)
$$

Note that this is equivalent to the cosine of the angle between $\vec{C}_{V}$ and $\vec{C}_{R}$. Using these definitions, rewrite equations 14 as

$$
\begin{aligned}
D & =\vec{C}_{V} \cdot \vec{C}_{V}=C_{V}^{2} \\
E & =\vec{C}_{R} \cdot \vec{C}_{V}=C_{R} C_{V} \Phi \\
F & =\vec{C}_{R} \cdot \vec{C}_{R}=C_{R}^{2}
\end{aligned}
$$

\section{Integration Constants for Optimal Trajectories}

The integration constants $\left(P_{i}, G_{i}, K_{i}\right)$ for each possible trajectory are defined using Thrust Plane notation.

VII.A. One-Phase Solution: $a_{T}=$ constant

$P, G$, and $K$ for a solution with one thrust acceleration subarc are given by: ${ }^{24}$

$$
\begin{aligned}
& P_{1}=a_{T}\left[C_{V}-\sqrt{C_{V}{ }^{2}+2 C_{R} C_{V} \Phi \tau_{o}+C_{R}{ }^{2} \tau_{o}^{2}}\right] \\
& G_{1}=-a_{T} \ln \left[\frac{\sqrt{C_{V}^{2}+2 C_{R} C_{V} \Phi \tau_{o}+C_{R}^{2} \tau_{o}^{2}}+C_{R} \tau_{o}+C_{V} \Phi}{C_{V}+C_{V} \Phi}\right] \\
& K_{1}=\frac{1}{2} a_{T} \tau_{o} \sqrt{C_{V}^{2}+2 C_{R} C_{V} \Phi \tau_{o}+C_{R}^{2} \tau_{o}^{2}}
\end{aligned}
$$

VII.B. Two-Phase Solution: $a_{T_{\text {not }}}=a_{T_{\max }}, a_{T_{\text {fin }}}=a_{T_{\min }}$

$P, G$, and $K$ for a solution with two thrust acceleration subarcs with $a_{T_{\text {not }}}=a_{T_{\max }}, a_{T_{\text {fin }}}=a_{T_{\min }}$ are given by: ${ }^{24}$ 


$$
\begin{aligned}
P_{2_{\max } \min } & =a_{T_{\min }}\left(C_{V}-1\right)-a_{T_{\max }}\left(\sqrt{C_{V}^{2}+2 C_{R} C_{V} \Phi \tau_{o}+C_{R}^{2} \tau_{o}^{2}}-1\right) \\
G_{2_{\max -\min }} & =-\ln \left[\left(\frac{1+\sqrt{C_{V}^{2} \Phi^{2}-\left(C_{V}^{2}-1\right)}}{C_{V}+C_{V} \Phi}\right)^{a_{T_{\min }}}\right] \\
& -\ln \left[\left(\frac{\sqrt{C_{V}^{2}+2 C_{R} C_{V} \Phi \tau_{o}+C_{R}^{2} \tau_{o}^{2}}+C_{R} \tau_{o}+C_{V} \Phi}{1+\sqrt{C_{V}^{2} \Phi^{2}-\left(C_{V}^{2}-1\right)}}\right)^{a_{T_{\max }}}\right] \\
K_{2_{\max -\min }} & =\frac{1}{2}\left(a_{T_{\min }}-a_{T_{\max }}\right)\left(\frac{-C_{V} \Phi+\sqrt{C_{V}^{2} \Phi^{2}-\left(C_{V}^{2}-1\right)}}{C_{R}}\right) \\
& +\frac{1}{2} a_{T_{\max }} \tau_{o} \sqrt{C_{V}^{2}+2 C_{R} C_{V} \Phi \tau_{o}+C_{R}^{2} \tau_{o}^{2}}
\end{aligned}
$$

VII.C. Two-Phase Solution: $a_{T_{\text {not }}}=a_{T_{\min }}, a_{T_{\text {fin }}}=a_{T_{\max }}$

$P, G$, and $K$ for a solution with two thrust acceleration subarcs with $a_{T_{n o t}}=a_{T_{\min }}, a_{T_{f i n}}=a_{T_{\max }}$ are given by: ${ }^{24}$

$$
\begin{aligned}
P_{2_{\min -\max }} & =\left[a_{T_{\max }}\left(C_{V}-1\right)-a_{T_{\min }}\left(\sqrt{C_{V}^{2}+2 C_{R} C_{V} \Phi \tau_{o}+C_{R}^{2} \tau_{o}^{2}}-1\right)\right] \\
G_{2_{\min -\max }} & =-\ln \left[\left(\frac{1-\sqrt{C_{V}^{2} \Phi^{2}-\left(C_{V}^{2}-1\right)}}{C_{V}+C_{V} \Phi}\right)^{a_{T_{\max }}}\right] \\
& -\ln \left[\left(\frac{\sqrt{C_{V}^{2}+2 C_{R} C_{V} \Phi \tau_{o}+C_{R}^{2} \tau_{o}^{2}}+C_{R} \tau_{o}+C_{V} \Phi}{1-\sqrt{C_{V}^{2} \Phi^{2}-\left(C_{V}^{2}-1\right)}}\right)^{a_{T_{\min }}}\right] \\
K_{2_{\min -\max }} & =\frac{1}{2}\left(a_{T_{\max }}-a_{T_{\min }}\right)\left(\frac{-C_{V} \Phi-\sqrt{C_{V}^{2} \Phi^{2}-\left(C_{V}^{2}-1\right)}}{C_{R}}\right) \\
& +\frac{1}{2} a_{T_{\min }} \tau_{o} \sqrt{C_{V}^{2}+2 C_{R} C_{V} \Phi \tau_{o}+C_{R}^{2} \tau_{o}^{2}}
\end{aligned}
$$

\section{VII.D. Three-Phase Solution}

$P, G$, and $K$ for a solution with three thrust acceleration subarcs are given by: ${ }^{24}$

$$
\begin{aligned}
& P_{3}=a_{T_{\max }}\left[C_{V}-\sqrt{C_{V}{ }^{2}+2 C_{R} C_{V} \Phi \tau_{o}+C_{R}{ }^{2} \tau_{o}^{2}}\right] \\
& G_{3}=-\ln \left[\left(\frac{\sqrt{C_{V}^{2}+2 C_{R} C_{V} \Phi \tau_{o}+C_{R}^{2} \tau_{o}^{2}}+C_{R} \tau_{o}+C_{V} \Phi}{C_{V}+C_{V} \Phi}\right)^{a_{T_{\max }}}\right] \\
& -\ln \left[\left(\frac{1-\sqrt{C_{V}^{2} \Phi^{2}-\left(C_{V}^{2}-1\right)}}{1+\sqrt{C_{V}^{2} \Phi^{2}-\left(C_{V}^{2}-1\right)}}\right)^{\left(a_{T_{\max }}-a_{T_{\min }}\right)}\right] \\
& K_{3}=\frac{1}{2} a_{T_{\max }} \tau_{o} \sqrt{C_{V}^{2}+2 C_{R} C_{V} \Phi \tau_{o}+C_{R}^{2} \tau_{o}^{2}} \\
& -\left(a_{T_{\max }}-a_{T_{\min }}\right) \frac{\sqrt{C_{V}^{2} \Phi^{2}-\left(C_{V}^{2}-1\right)}}{C_{R}}
\end{aligned}
$$




\section{Coefficient Relationships}

Starting with equations 27 and applying various combinations of vector dot products and vector cross products with $\vec{C}_{R}, \vec{C}_{V}, \tilde{R}$, and $\tilde{V}$, it is possible to derive the following four relationships.

$$
\begin{aligned}
P & =\left(\vec{C}_{R} \cdot \tilde{V}\right) \\
G & =\frac{\sqrt{F}\left[F\left(\vec{C}_{V} \cdot \tilde{V}\right)-E\left(\vec{C}_{R} \cdot \tilde{V}\right)\right]}{\left(D F-E^{2}\right)} \\
K & =\left[-\left(\vec{C}_{R} \cdot \tilde{R}\right)-\frac{1}{2}\left(\vec{C}_{V} \cdot \tilde{V}\right)\right] \\
0 & =\left(\vec{C}_{R} \times \tilde{R}\right)+\left(\vec{C}_{V} \times \tilde{V}\right)
\end{aligned}
$$

The derivations are straight-forward but cumbersome. For details, see Appendix $\mathrm{C}$ of reference 24 .

Equations 37 represent a system of four equations in five unknowns $\left(C_{V}, C_{R}, \theta_{C_{V}}, \theta_{C_{R}}\right.$, and $\left.\tau_{o}\right)$. The first of equations 37 is equivalent to the condition that the Hamiltonian is constant along the optimal trajectory from equation 23. It can be derived by subtracting the final value of the Hamiltonian from the initial value of the Hamiltonian. The fourth of equations 37 is equivalent to the condition of rotational symmetry from equation 25. It can be derived by subtracting the condition applied at the initial time from the condition applied to the final time. The fifth equation to complete the system comes from the knowledge that the Hamiltonian has a value of zero. It will be convenient to apply this condition at the final time.

$$
H_{f}=a_{T_{f}}[1-\sqrt{D}]+\vec{C}_{R} \cdot \vec{V}_{f}+\vec{C}_{V} \cdot \vec{g}=0
$$

In Thrust Plane notation, equations 37 become

$$
\begin{aligned}
P & =C_{R} \tilde{V}_{\text {mag }}\left(\cos \theta_{C_{R}} \cos \theta_{\tilde{V}}+\sin \theta_{C_{R}} \sin \theta_{\tilde{V}}\right) \\
G & =\frac{C_{R}}{C_{V}} \tilde{V}_{\text {mag }} \frac{\left(\cos \theta_{C_{R}} \sin \theta_{\tilde{V}}-\sin \theta_{C_{R}} \cos \theta_{\tilde{V}}\right)}{\left(\cos \theta_{C_{R}} \sin \theta_{C_{V}}-\sin \theta_{C_{R}} \cos \theta_{C_{V}}\right)} \\
K & =-C_{R} \tilde{R}_{\text {mag }}\left(\cos \theta_{C_{R}} \cos \theta_{\tilde{R}}+\sin \theta_{C_{R}} \sin \theta_{\tilde{R}}\right) \\
& -\frac{1}{2} C_{V} \tilde{V}_{\text {mag }}\left(\cos \theta_{C_{V}} \cos \theta_{\tilde{V}}+\sin \theta_{C_{V}} \sin \theta_{\tilde{V}}\right) \\
0 & =C_{R} \tilde{R}_{\text {mag }}\left(\cos \theta_{C_{R}} \sin \theta_{\tilde{R}}-\sin \theta_{C_{R}} \cos \theta_{\tilde{R}}\right) \\
& +C_{V} \tilde{V}_{\text {mag }}\left(\cos \theta_{C_{V}} \sin \theta_{\tilde{V}}-\sin \theta_{C_{V}} \cos \theta_{\tilde{V}}\right)
\end{aligned}
$$

and the final Hamiltonian condition is

$$
\begin{aligned}
0 & =a_{T_{f}}\left[1-C_{V}\right] \\
& +C_{R} V_{f} \cos \phi_{V_{f}}\left(\cos \theta_{C_{R}} \cos \theta_{V_{f}}+\sin \theta_{C_{R}} \sin \theta_{V_{f}}\right) \\
& +C_{V} g \cos \phi_{g}\left(\cos \theta_{C_{V}} \cos \theta_{g}+\sin \theta_{C_{V}} \sin \theta_{g}\right)=0
\end{aligned}
$$

Equations 39 through 43 represent a system of five equations in five unknowns: $C_{V}, C_{R}, \theta_{C_{V}}, \theta_{C_{R}}$, and $\tau_{o}$.

\section{Reduction of Optimal Problem to Two Variables}

It is possible to analytically reduce the optimal problem to two variables. For these derivations, it is assumed that a constant thrust direction solution is not valid. This is equivalent to the assumption that all of the vectors $\vec{C}_{R}, \vec{C}_{V}, \tilde{R}$, and $\tilde{V}$ are non-zero and that none of them are parallel. Recall that the Thrust Plane Coordinate system X-axis was defined as along the direction of $\tilde{V}$. Thus, the angle $\theta_{\tilde{V}}$ is equal to zero by definition. However, for purposes of a general derivation, this fact will not be used. The first step is to

solve equations 42 and 43 for $C_{R}$ and $C_{V}$ in terms of $\theta_{C_{R}}, \theta_{C_{V}}$, and $\tau_{o}$. Start with equation 42 and solve for $C_{R}$ to obtain

$$
C_{R}=-C_{V} \frac{\tilde{V}_{\text {mag }}\left(\cos \theta_{C_{V}} \sin \theta_{\tilde{V}}-\sin \theta_{C_{V}} \cos \theta_{\tilde{V}}\right)}{\tilde{R}_{\text {mag }}\left(\cos \theta_{C_{R}} \sin \theta_{\tilde{R}}-\sin \theta_{C_{R}} \cos \theta_{\tilde{R}}\right)}
$$


Now take the final Hamiltonian in equation 43 and solve for $C_{R}$

$$
C_{R}=-\frac{a_{T_{f}}\left[1-C_{V}\right]+C_{V} g \cos \phi_{g}\left[\cos \theta_{C_{V}} \cos \theta_{g}+\sin \theta_{C_{V}} \sin \theta_{g}\right]}{V_{f} \cos \phi_{V_{f}}\left[\cos \theta_{C_{R}} \cos \theta_{V_{f}}+\sin \theta_{C_{R}} \sin \theta_{V_{f}}\right]}
$$

Set equations 44 and 45 equal and solve for $C_{V}$, leading to

$$
C_{V}=\frac{a_{T_{f}} \tilde{R}_{m a g}\left(\sin \theta_{\tilde{R}}-\tan \theta_{C_{R}} \cos \theta_{\tilde{R}}\right)}{\left(C T V_{1} \tan \theta_{C_{R}}+C T V_{2}\right)}
$$

where

$$
\begin{aligned}
C T V_{1} & =\left(C T_{1} \cos \theta_{C_{V}}+C T_{2} \sin \theta_{C_{V}}+C T_{3}\right) \\
C T V_{2} & =\left(C T_{4} \cos \theta_{C_{V}}+C T_{5} \sin \theta_{C_{V}}+C T_{6}\right) \\
C T_{1} & =\tilde{V}_{m a g} V_{f} \cos \phi_{V_{f}} \sin \theta_{V_{f}} \sin \theta_{\tilde{V}}+\tilde{R}_{m a g} g \cos \phi_{g} \cos \theta_{g} \cos \theta_{\tilde{R}} \\
C T_{2} & =-\tilde{V}_{m a g} V_{f} \cos \phi_{V_{f}} \sin \theta_{V_{f}} \cos \theta_{\tilde{V}}+\tilde{R}_{m a g} g \cos \phi_{g} \sin \theta_{g} \cos \theta_{\tilde{R}} \\
C T_{3} & =-a_{T_{f}} \tilde{R}_{m a g} \cos \theta_{\tilde{R}} \\
C T_{4} & =\tilde{V}_{m a g} V_{f} \cos \phi_{V_{f}} \cos \theta_{V_{f}} \sin \theta_{\tilde{V}}-\tilde{R}_{m a g} g \cos \phi_{g} \cos \theta_{g} \sin \theta_{\tilde{R}} \\
C T_{5} & =-\tilde{V}_{\text {mag }} V_{f} \cos \phi_{V_{f}} \cos \theta_{V_{f}} \cos \theta_{\tilde{V}}-\tilde{R}_{m a g} g \cos \phi_{g} \sin \theta_{g} \sin \theta_{\tilde{R}} \\
C T_{6} & =a_{T_{f}} \tilde{R}_{m a g} \sin \theta_{\tilde{R}}
\end{aligned}
$$

Now substitute equation 46 into equation 44 to find $C_{R}$, resulting in

$$
C_{R}=-\frac{a_{T_{f}} \tilde{V}_{m a g}\left(\sin \theta_{\tilde{V}} \cos \theta_{C_{V}}-\cos \theta_{\tilde{V}} \sin \theta_{C_{V}}\right)}{\cos \theta_{C_{R}}\left(C T V_{1} \tan \theta_{C_{R}}+C T V_{2}\right)}
$$

Define the following variables:

$$
\begin{aligned}
C T V_{3} & =C T_{7} \cos \theta_{C_{V}}+C T_{8} \sin \theta_{C_{V}} \\
C T_{7} & =-a_{T_{f}} \tilde{V}_{\text {mag }} \sin \theta_{\tilde{V}} \\
C T_{8} & =a_{T_{f}} \tilde{V}_{\text {mag }} \cos \theta_{\tilde{V}} \\
C T_{9} & =-a_{T_{f}} \tilde{R}_{\text {mag }} \cos \theta_{\tilde{R}} \\
C T_{10} & =a_{T_{f}} \tilde{R}_{\text {mag }} \sin \theta_{\tilde{R}}
\end{aligned}
$$

Equations 46 and 48 can be written as:

$$
\begin{aligned}
C_{R} & =\frac{C T V_{3}}{\cos \theta_{C_{R}}\left(C T V_{1} \tan \theta_{C_{R}}+C T V_{2}\right)} \\
C_{V} & =\frac{C T_{9} \tan \theta_{C_{R}}+C T_{10}}{C T V_{1} \tan \theta_{C_{R}}+C T V_{2}}
\end{aligned}
$$

Comparison of $P_{i}$ from equations 33, 34, 35, and 36 shows that a general equation for $P$ can be written as:

$$
P=\left(\vec{C}_{R} \cdot \tilde{V}\right)=a_{T_{f}}(\sqrt{D}-1)-a_{T_{o}}\left(\sqrt{D+2 E \tau_{o}+F \tau_{o}^{2}}-1\right)
$$

In Thrust Plane notation, this equation becomes

$$
\begin{aligned}
& C_{R} \tilde{V}_{\text {mag }}\left(\cos \theta_{C_{R}} \cos \theta_{\tilde{V}}+\sin \theta_{C_{R}} \sin \theta_{\tilde{V}}\right) \\
= & a_{T_{f}}\left(C_{V}-1\right)-a_{T_{o}}\left(\sqrt{C_{V}^{2}+2 C_{R} C_{V} \Phi \tau_{o}+C_{R}^{2} \tau_{o}^{2}}-1\right)
\end{aligned}
$$


Substitute equation 31 for $\Phi$ into equation 52 , isolate the square root term, square each side, and collect terms multiplied by $C_{R}$ and $C_{V}$. After some manipulation, we obtain

$$
\begin{aligned}
0= & {\left[\tilde{V}_{\text {mag }}^{2}\left(\cos \theta_{\tilde{V}}+\sin \theta_{\tilde{V}} \tan \theta_{C_{R}}\right)^{2}-\frac{a_{T_{o}}^{2} \tau_{o}^{2}}{\cos ^{2} \theta_{C_{R}}}\right] \cos ^{2} \theta_{C_{R}} C_{R}^{2} } \\
+ & \left(a_{T_{f}}^{2}-a_{T_{o}}^{2}\right) C_{V}^{2} \\
- & {\left[2 a_{T_{f}} \tilde{V}_{\text {mag }}\left(\cos \theta_{\tilde{V}}+\sin \theta_{\tilde{V}} \tan \theta_{C_{R}}\right) \ldots\right.} \\
& \left.\quad+2 a_{T_{o}}^{2}\left(\cos \theta_{C_{V}}+\sin \theta_{C_{V}} \tan \theta_{C_{R}}\right) \tau_{o}\right] \cos \theta_{C_{R}} C_{R} C_{V} \\
+ & 2\left(a_{T_{f}}-a_{T_{o}}\right) \tilde{V}_{\text {mag }}\left(\cos \theta_{\tilde{V}}+\sin \theta_{\tilde{V}} \tan \theta_{C_{R}}\right) \cos \theta_{C_{R}} C_{R} \\
- & 2 a_{T_{f}}\left(a_{T_{f}}-a_{T_{o}}\right) C_{V} \\
+ & \left(a_{T_{f}}-a_{T_{o}}\right)^{2}
\end{aligned}
$$

Note the following trigonometric identity: ${ }^{42}$

$$
\frac{1}{\cos ^{2} \theta_{C_{R}}}=\left(1+\tan ^{2} \theta_{C_{R}}\right)
$$

After substituting this identity and some manipulation, equation 53 can be written as

$$
\begin{aligned}
0 & =\left(C A_{1} \tan ^{2} \theta_{C_{R}}+C A_{2} \tan \theta_{C_{R}}+C A_{3}\right) \cos ^{2} \theta_{C_{R}} C_{R}^{2} \\
& +\left(C T V_{4} \tan \theta_{C_{R}}+C T V_{5}\right) \cos \theta_{C_{R}} C_{R} C_{V} \\
& +\left(C C_{1} \tan \theta_{C_{R}}+C C_{2}\right) \cos \theta_{C_{R}} C_{R} \\
& +C D_{1} C_{V}^{2}+C D_{2} C_{V}+C D_{3}
\end{aligned}
$$

where

$$
\begin{aligned}
C T V_{4} & =\left(C B_{1} \sin \theta_{C_{V}}+C B_{2}\right) \\
C T V_{5} & =\left(C B_{1} \cos \theta_{C_{V}}+C B_{3}\right) \\
C A_{1} & =\tilde{V}_{m a g}^{2} \sin ^{2} \theta_{\tilde{V}}-a_{T_{o}}^{2} \tau_{o}^{2} \\
C A_{2} & =2 \tilde{V}_{m a g}^{2} \sin \theta_{\tilde{V}} \cos \theta_{\tilde{V}} \\
C A_{3} & =\tilde{V}_{m a g}^{2} \cos ^{2} \theta_{\tilde{V}}-a_{T_{o}}^{2} \tau_{o}^{2} \\
C B_{1} & =-2 a_{T_{o}}^{2} \tau_{o} \\
C B_{2} & =-2 a_{T_{f}} \tilde{V}_{m a g} \sin \theta_{\tilde{V}} \\
C B_{3} & =-2 a_{T_{f}} \tilde{V}_{m a g} \cos \theta_{\tilde{V}} \\
C C_{1} & =2\left(a_{T_{f}}-a_{T_{o}}\right) \tilde{V}_{m a g} \sin \theta_{\tilde{V}} \\
C C_{2} & =2\left(a_{T_{f}}-a_{T_{o}}\right) \tilde{V}_{m a g} \cos \theta_{\tilde{V}} \\
C D_{1} & =\left(a_{T_{f}}^{2}-a_{T_{o}}^{2}\right) \\
C D_{2} & =-2 a_{T_{f}}\left(a_{T_{f}}-a_{T_{o}}\right) \\
C D_{3} & =\left(a_{T_{f}}-a_{T_{o}}\right)^{2}
\end{aligned}
$$

The next step is to substitute $C_{R}$ and $C_{V}$ from equation 50 into equation 55. After some manipulation, a quadratic equation for $\tan \theta_{C_{R}}$ can be written as

$$
0=C_{T C R 2} \tan ^{2} \theta_{C_{R}}+C_{T C R 1} \tan \theta_{C_{R}}+C_{T C R 0}
$$


where

$$
\begin{aligned}
C_{T C R 2} & =C T V_{3}^{2} C A_{1}+C T V_{1}^{2} C D_{3} \\
& +C T V_{1} C T V_{3} C C_{1}+C T V_{3} C T V_{4} C T_{9} \\
& +C T V_{1} C D_{2} C T_{9}+C D_{1} C T_{9}^{2} \\
C_{T C R 1} & =C T V_{3}^{2} C A_{2}+C T V_{3}\left(C T V_{4} C T_{10}+C T V_{5} C T_{9}\right) \\
& +C T V_{1}\left(C D_{2} C T_{10}+C T V_{2} C D_{3}+C T V_{3} C C_{2}\right) \\
& +C T V_{2}\left(C D_{2} C T_{9}+C T V_{1} C D_{3}+C T V_{3} C C_{1}\right) \\
& +2 C D_{1} C T_{9} C T_{10} \\
C_{T C R 0} & =C T V_{3}^{2} C A_{3}+C T V_{2}^{2} C D_{3} \\
& +C T V_{2} C T V_{3} C C_{2}+C T V_{3} C T V_{5} C T_{10} \\
& +C T V_{2} C D_{2} C T_{10}+C D_{1} C T_{10}^{2}
\end{aligned}
$$

Note that the coefficients in equations 58 are functions of $\theta_{C_{V}}$ and $\tau_{o}$.

A numerical search can be performed to solve for the values of $\theta_{C_{V}}$ and $\tau_{o}$. For a given value of $\theta_{C_{V}}$ and $\tau_{o}$, first assume a thrust sequence in order to set the initial and final values of the thrust acceleration magnitude. Now, compute all the values of $C A_{i}, C B_{i}, C C_{i}$, and $C D_{i}$ from equations 56. Also, compute the values of the $C T_{i}$ coefficients from equations 47 and 49 . Note that all of these coefficients are only functions of $\tau_{o}$. Now compute the values of the $C T V_{i}$ coefficients defined in equations 47,49 , and 56 . The coefficients $C_{T C R 2}, C_{T C R 1}$, and $C_{T C R 0}$ defined in equation 58 can now be calculated. Two possible values for $\tan \theta_{C_{R}}$ can be computed from equation 57. From equation 54, two possible values of the absolute value of $\cos \theta_{C_{R}}$ are given by

$$
\left|\cos \theta_{C_{R}}\right|=\frac{1}{\sqrt{1+\tan ^{2} \theta_{C_{R}}}}
$$

Using the two possible values for $\left|\cos \theta_{C_{R}}\right|$ and $\tan \theta_{C_{R}}$, two possible values for $C_{R}$ and $C_{V}$ can be found from equations 50. The proper sign of $\cos \theta_{C_{R}}$ is selected using the fact that $C_{R}$ must be positive. With the values of $\tan \theta_{C_{R}}$ and $\cos \theta_{C_{R}}$, two possible values of $\theta_{C_{R}}$ can be found. There is now enough information to calculate two possible values for $\vec{C}_{R}$ and $\vec{C}_{V}$. The values of the integration constants $P, G$, and $K$ can be computed from the equations defined in section VII. The $\tilde{V}$ and $\tilde{R}$ vectors can be computed from equations 27. Given the initial position, initial velocity, and gravity, the values of the final position and velocity can be computed from equation 26 . The problem then reduces to finding the values of $\theta_{C_{V}}$ and $\tau_{o}$ that meet the desired final position and velocity. The variable $\theta_{C_{V}}$ is bounded because it is an angle. It will be shown in section XI that the value of $\tau_{o}$ can be bounded analytically. Thus, the problem is reduced to a two-dimensional bounded search.

\section{Reduction of One-Phase Optimal Problem to One Variable}

When the problem is constrained to a constant thrust acceleration magnitude, it is possible to analytically reduce the optimal problem to one variable. As in the previous section, it is assumed that a constant thrust direction solution is not valid. This is equivalent to the assumption that all of the vectors $\vec{C}_{R}, \vec{C}_{V}, \tilde{R}$, and $\tilde{V}$ are non-zero and that none of them are parallel. For a constant thrust acceleration problem, the definitions of $P_{1}, G_{1}$, and $K_{1}$ are given in equations 33. Set $P_{1}=P, G_{1}=G, K_{1}=K$ in equations 39 through 41, respectively. Define the ratio of $C_{R}$ to $C_{V}$ as

$$
\rho=\frac{C_{R}}{C_{V}}
$$

Rearrange equations 39 through 43 in terms of $\rho$. Recall that the angle $\theta_{\tilde{V}}$ is equal to zero by definition.

$$
\begin{gathered}
a_{T}\left[1-\sqrt{1+2 \rho \Phi \tau_{o}+\rho^{2} \tau_{o}^{2}}\right]=\rho \tilde{V}_{\text {mag }} \cos \theta_{C_{R}} \\
a_{T} \ln \left[\frac{\sqrt{1+2 \rho \Phi \tau_{o}+\rho^{2} \tau_{o}^{2}}+\rho \tau_{o}+\Phi}{1+\Phi}\right] \\
=\frac{\rho \tilde{V}_{\text {mag }} \sin \theta_{C_{R}}}{\left(\cos \theta_{C_{R}} \sin \theta_{C_{V}}-\sin \theta_{C_{R}} \cos \theta_{C_{V}}\right)}
\end{gathered}
$$




$$
\begin{aligned}
& \frac{1}{2} a_{T} \tau_{o} \sqrt{1+2 \rho \Phi \tau_{o}+\rho^{2} \tau_{o}^{2}} \\
&=-\rho \tilde{R}_{\text {mag }}\left(\cos \theta_{C_{R}} \cos \theta_{\tilde{R}}+\sin \theta_{C_{R}} \sin \theta_{\tilde{R}}\right)-\frac{1}{2} \tilde{V}_{\text {mag }} \cos \theta_{C_{V}} \\
& 0= \rho \tilde{R}_{m a g}\left(\cos \theta_{C_{R}} \sin \theta_{\tilde{R}}-\sin \theta_{C_{R}} \cos \theta_{\tilde{R}}\right)-\tilde{V}_{\text {mag }} \sin \theta_{C_{V}} \\
& 0= a_{T_{f}}\left[\frac{1}{C_{V}}-1\right] \\
& \quad+\quad \rho V_{f} \cos \phi_{V_{f}}\left(\cos \theta_{C_{R}} \cos \theta_{V_{f}}+\sin \theta_{C_{R}} \sin \theta_{V_{f}}\right) \\
& \quad+g \cos \phi_{g}\left(\cos \theta_{C_{V}} \cos \theta_{g}+\sin \theta_{C_{V}} \sin \theta_{g}\right)
\end{aligned}
$$

Equations 61 through 64 represent a system of four equations in four unknowns: $\rho, \theta_{C_{R}}$, $\theta_{C_{V}}$, and $\tau_{o}$. The variable $C_{V}$ can be freely chosen to meet the condition in equation 65 , but its value is not needed to solve the system of equations. Fill noted this fact: for a constant thrust or constant acceleration problem, the dimension can be reduced by "scaling the primer" vector ${ }^{12}$ (p. 19). Multiply equation 61 by $\frac{1}{2} \tau_{o}$ and rearrange to obtain

$$
\frac{1}{2} a_{T} \tau_{o} \sqrt{1+2 \rho \Phi \tau_{o}+\rho^{2} \tau_{o}^{2}}=\frac{1}{2} \tau_{o}\left[a_{T}-\rho \tilde{V}_{m a g} \cos \theta_{C_{R}}\right]
$$

Setting the right-hand side of equation 66 equal to the right-hand side of equation 63 yields

$$
\begin{aligned}
& \frac{1}{2} \tau_{o}\left[a_{T}-\rho \tilde{V}_{\text {mag }} \cos \theta_{C_{R}}\right] \\
= & -\rho \tilde{R}_{\text {mag }}\left(\cos \theta_{C_{R}} \cos \theta_{\tilde{R}}+\sin \theta_{C_{R}} \sin \theta_{\tilde{R}}\right)-\frac{1}{2} \tilde{V}_{\text {mag }} \cos \theta_{C_{V}}
\end{aligned}
$$

Solving equation 67 for $\rho$ and writing it in terms of $\tan \theta_{C_{R}}$ results in

$$
\rho=\frac{\left[\tilde{V}_{\text {mag }} \cos \theta_{C_{V}}+a_{T} \tau_{o}\right]}{\cos \theta_{C_{R}}\left[\left(-2 \tilde{R}_{\text {mag }} \sin \theta_{\tilde{R}}\right) \tan \theta_{C_{R}}+\left(\tilde{V}_{\text {mag }} \tau_{o}-2 \tilde{R}_{\text {mag }} \cos \theta_{\tilde{R}}\right)\right]}
$$

Also solve equation 64 for $\rho$ and write it in terms of $\tan \theta_{C_{R}}$ to obtain

$$
\rho=\frac{\tilde{V}_{\text {mag }} \sin \theta_{C_{V}}}{\cos \theta_{C_{R}} \tilde{R}_{m a g}\left(\sin \theta_{\tilde{R}}-\tan \theta_{C_{R}} \cos \theta_{\tilde{R}}\right)}
$$

Setting equation 68 equal to equation 69 and rearranging yields

$$
\begin{aligned}
0 & =-\left[D_{C_{R}} \sin \theta_{C_{V}}+E_{C_{R}} \cos \theta_{C_{V}}+F_{C_{R}}\right] \tan \theta_{C_{R}} \cos \theta_{C_{R}} \\
& +\left[A_{C_{R}} \sin \theta_{C_{V}}+B_{C_{R}} \cos \theta_{C_{V}}+C_{C_{R}}\right] \cos \theta_{C_{R}}
\end{aligned}
$$

where

$$
\begin{aligned}
A_{C_{R}} & =2 \tilde{R}_{m a g} \tilde{V}_{\text {mag }} \cos \theta_{\tilde{R}}-\tilde{V}_{\text {mag }}^{2} \tau_{o} \\
B_{C_{R}} & =\tilde{R}_{m a g} \tilde{V}_{\text {mag }} \sin \theta_{\tilde{R}} \\
C_{C_{R}} & =a_{T} \tilde{R}_{\text {mag }} \tau_{o} \sin \theta_{\tilde{R}} \\
D_{C_{R}} & =-2 \tilde{R}_{m a g} \tilde{V}_{m a g} \sin \theta_{\tilde{R}} \\
E_{C_{R}} & =\tilde{R}_{\text {mag }} \tilde{V}_{\text {mag }} \cos \theta_{\tilde{R}} \\
F_{C_{R}} & =a_{T} \tilde{R}_{m a g} \tau_{o} \cos \theta_{\tilde{R}}
\end{aligned}
$$

One possible solution is $\cos \theta_{C_{R}}=0$. However, from equation 61 this would mean that $S S=\sqrt{1+2 \rho \Phi \tau_{o}+\rho^{2} \tau_{o}^{2}}=$ 1. This is equivalent to the special case when an off boundary thrust acceleration can solve the problem. This leads to a constant thrust direction solution. It was assumed at the beginning of the section that this case is not valid for the current derivation. Thus, for $\cos \theta_{C_{R}} \neq 0$ we have

$$
\tan \theta_{C_{R}}=\frac{A_{C_{R}} \sin \theta_{C_{V}}+B_{C_{R}} \cos \theta_{C_{V}}+C_{C_{R}}}{D_{C_{R}} \sin \theta_{C_{V}}+E_{C_{R}} \cos \theta_{C_{V}}+F_{C_{R}}}
$$


Now return to equation 61 . Isolate the square root and square both sides to obtain

$$
0=\left(2 a_{T}^{2} \Phi \tau_{o}+2 a_{T} \tilde{V}_{m a g} \cos \theta_{C_{R}}\right) \rho+\left(a_{T}^{2} \tau_{o}^{2}-\tilde{V}_{m a g}^{2} \cos ^{2} \theta_{C_{R}}\right) \rho^{2}
$$

One possible solution to this equation is $\rho=0$. This implies that $\vec{C}_{R}=0$ and leads to a constant unit thrust direction with an off-boundary thrust acceleration magnitude. This solution is equivalent to dropping the constraint on final position. Thus, it is a special case that can be checked. Thus, for $\rho \neq 0$ we have

$$
\rho=-\left[\frac{2 a_{T}^{2} \Phi \tau_{o}+2 a_{T} \tilde{V}_{m a g} \cos \theta_{C_{R}}}{a_{T}^{2} \tau_{o}^{2}-\tilde{V}_{m a g}^{2} \cos ^{2} \theta_{C_{R}}}\right]
$$

Set equation 74 equal to equation 69 , substitute for the definition of $\Phi$ from equation 31, use the trigonometric identity in equation 54 , and rearrange to find a quadratic equation in $\tan \theta_{C_{R}}$.

$$
\begin{aligned}
0 & =\left[C_{S 0} \sin \theta_{C_{V}}+C_{C 0} \cos \theta_{C_{V}}+C_{0}\right] \\
& +\left[C_{S 1} \sin \theta_{C_{V}}+C_{C 1} \cos \theta_{C_{V}}+C_{1}\right] \tan \theta_{C_{R}} \\
& +\left[C_{S 2} \sin \theta_{C_{V}}\right] \tan ^{2} \theta_{C_{R}}
\end{aligned}
$$

where

$$
\begin{aligned}
C_{S 0} & =a_{T}^{2} \tau_{o}^{2} \tilde{V}_{m a g}-\tilde{V}_{m a g}^{3} \\
C_{C 0} & =2 a_{T}^{2} \tau_{o} \tilde{R}_{m a g} \sin \theta_{\tilde{R}} \\
C_{0} & =2 a_{T} \tilde{R}_{m a g} \tilde{V}_{m a g} \sin \theta_{\tilde{R}} \\
C_{S 1} & =2 a_{T}^{2} \tau_{o} \tilde{R}_{m a g} \sin \theta_{\tilde{R}} \\
C_{C 1} & =-2 a_{T}^{2} \tau_{o} \tilde{R}_{m a g} \cos \theta_{\tilde{R}} \\
C_{1} & =-2 a_{T} \tilde{R}_{m a g} \tilde{V}_{m a g} \cos \theta_{\tilde{R}} \\
C_{S 2} & =a_{T}^{2} \tau_{o}^{2} \tilde{V}_{m a g}-2 a_{T}^{2} \tau_{o} \tilde{R}_{m a g} \cos \theta_{\tilde{R}}
\end{aligned}
$$

Now, substitute $\tan \theta_{C_{R}}$ from equation 72 into equation 75 to obtain

$$
\begin{aligned}
& D_{S^{3} C^{0}} \sin ^{3} \theta_{C_{V}} \\
& +D_{S^{0} C^{3} \cos ^{3} \theta_{C_{V}}}
\end{aligned}
$$

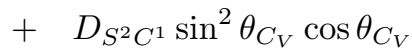

$$
\begin{aligned}
& +D_{S^{1} C^{2}} \sin \theta_{C_{V}} \cos ^{2} \theta_{C_{V}} \\
& +D_{S^{2} C^{0}} \sin ^{2} \theta_{C_{V}} \\
& +D_{S^{0} C^{2} \cos ^{2} \theta_{C_{V}}} \\
& +D_{S^{1} C^{1}} \sin \theta_{C_{V}} \cos \theta_{C_{V}} \\
& +D_{S^{1} C^{0}} \sin \theta_{C_{V}} \\
& +D_{S^{0} C^{1}} \cos \theta_{C_{V}} \\
& +D_{S^{0} C^{0}}=0
\end{aligned}
$$


where

$$
\begin{aligned}
D_{S^{3} C^{0}} & =C_{S 0} D_{C_{R}}^{2}+C_{S 1} A_{C_{R}} D_{C_{R}}+C_{S 2} A_{C_{R}}^{2} \\
D_{S^{0} C^{3}} & =C_{C 0} E_{C_{R}}^{2}+C_{C 1} B_{C_{R}} E_{C_{R}} \\
D_{S^{2} C^{1}} & =C_{C 0} D_{C_{R}}^{2}+2 C_{S 0} D_{C_{R}} E_{C_{R}}+C_{C 1} A_{C_{R}} D_{C_{R}} \\
& +C_{S 1}\left(A_{C_{R}} E_{C_{R}}+B_{C_{R}} D_{C_{R}}\right)+2 C_{S 2} A_{C_{R}} B_{C_{R}} \\
D_{S^{1} C^{2}} & =C_{S 0} E_{C_{R}}^{2}+2 C_{C 0} D_{C_{R}} E_{C_{R}}+C_{S 1} B_{C_{R}} E_{C_{R}} \\
& +C_{C 1}\left(A_{C_{R}} E_{C_{R}}+B_{C_{R}} D_{C_{R}}\right)+C_{S 2} B_{C_{R}}^{2} \\
D_{S^{2} C^{0}} & =C_{0} D_{C_{R}}^{2}+2 C_{S 0} D_{C_{R}} F_{C_{R}}+C_{1} A_{C_{R}} D_{C_{R}} \\
& +C_{S 1}\left(A_{C_{R}} F_{C_{R}}+C_{C_{R}} D_{C_{R}}\right)+2 C_{S 2} A_{C_{R}} C_{C_{R}} \\
D_{S^{0} C^{2}} & =C_{0} E_{C_{R}}^{2}+2 C_{C 0} E_{C_{R}} F_{C_{R}}+C_{1} B_{C_{R}} E_{C_{R}} \\
& +C_{C_{1}}\left(B_{C_{R}} F_{C_{R}}+C_{C_{R}} E_{C_{R}}\right) \\
D_{S^{1} C^{1}} & =2 C_{0} D_{C_{R}} E_{C_{R}}+2 C_{C_{0}} D_{C_{R}} F_{C_{R}}+2 C_{S 0} E_{C_{R}} F_{C_{R}} \\
& +C_{1}\left(A_{C_{R}} E_{C_{R}}+B_{C_{R}} D_{C_{R}}\right)+C_{C 1}\left(A_{C_{R}} F_{C_{R}}+C_{C_{R}} D_{C_{R}}\right) \\
& +C_{S 1}\left(B_{C_{R}} F_{C_{R}}+C_{C_{R}} E_{C_{R}}\right)+2 C_{S 2} B_{C_{R}} C_{C_{R}} \\
D_{S^{1} C^{0}} & =C_{S 0} F_{C_{R}}^{2}+2 C_{0} D_{C_{R}} F_{C_{R}}+C_{1}\left(A_{C_{R}} F_{C_{R}}+C_{C_{R}} D_{C_{R}}\right) \\
& +C_{S 1} C_{C_{R}} F_{C_{R}}+C_{S_{2}} C_{C_{R}}^{2} \\
D_{S^{0} C^{1}} & =C_{C 0} F_{C_{R}}^{2}+2 C_{0} E_{C_{R}} F_{C_{R}}+C_{1}\left(B_{C_{R}} F_{C_{R}}+C_{C_{R}} E_{C_{R}}\right) \\
& +C_{C 1} C_{C_{R}} F_{C_{R}} \\
D_{S^{0} C^{0}} & =C_{0} F_{C_{R}}^{2}+C_{1} C_{C_{R}} F_{C_{R}}
\end{aligned}
$$

It is shown in reference 24 that all coefficients that do not multiply with a power of $\sin \theta_{C_{V}}$ are zero, so $D_{S^{0} C^{3}}=D_{S^{0} C^{2}}=D_{S^{0} C^{1}}=D_{S^{0} C^{0}}=0$. Equation 77 then reduces to

$$
\begin{aligned}
& D_{S^{3} C^{0}} \sin ^{3} \theta_{C_{V}} \\
+ & D_{S^{2} C^{1} \sin ^{2} \theta_{C_{V}} \cos \theta_{C_{V}}} \\
+ & D_{S^{1} C^{2}} \sin \theta_{C_{V}} \cos ^{2} \theta_{C_{V}} \\
+ & D_{S^{2} C^{0}} \sin ^{2} \theta_{C_{V}} \\
+ & D_{S^{1} C^{1} \sin \theta_{C_{V}} \cos \theta_{C_{V}}} \\
+ & D_{S^{1} C^{0} \sin \theta_{C_{V}}=0}
\end{aligned}
$$

One possible solution is $\sin \theta_{C_{V}}=0$. This means that $\vec{C}_{V}$ would be aligned with $\tilde{V}$. From equation 69 , it can be seen that $\rho=0$ when $\sin \theta_{C_{V}}=0$, and when $\rho=0$, then $\vec{C}_{R}=0$. As discussed previously, it has been assumed for purposes of this derivation that this is not the case. Thus, for $\sin \theta_{C_{V}} \neq 0$, equation 79 reduces further to

$$
\begin{aligned}
& D_{S^{3} C^{0} \sin ^{2} \theta_{C_{V}}} \\
+ & D_{S^{2} C^{1}} \sin \theta_{C_{V}} \cos \theta_{C_{V}} \\
+ & D_{S^{1} C^{2}} \cos ^{2} \theta_{C_{V}} \\
+ & D_{S^{2} C^{0} \sin \theta_{C_{V}}} \\
+ & D_{S^{1} C^{1}} \cos \theta_{C_{V}} \\
+ & D_{S^{1} C^{0}}=0
\end{aligned}
$$

Note the following well known trigonometric identity: ${ }^{42}$

$$
\begin{array}{ll} 
& \sin ^{2} \theta_{C_{V}}+\cos ^{2} \theta_{C_{V}}=1 \\
\Rightarrow & \cos ^{2} \theta_{C_{V}}=1-\sin ^{2} \theta_{C_{V}} \\
\Rightarrow & \cos \theta_{C_{V}}= \pm \sqrt{\left(1-\sin ^{2} \theta_{C_{V}}\right)}
\end{array}
$$


Use this identity in equation 80 , rearrange to isolate the square root term, square both sides, and rearrange:

$$
\begin{aligned}
& {\left[\left(D_{S^{3} C^{0}}-D_{S^{1} C^{2}}\right)^{2}+D_{S^{2} C^{1}}^{2}\right] \sin ^{4} \theta_{C_{V}} } \\
+ & {\left[2\left(D_{S^{3} C^{0}}-D_{S^{1} C^{2}}\right) D_{S^{2} C^{0}}+2 D_{S^{2} C^{1}} D_{S^{1} C^{1}}\right] \sin ^{3} \theta_{C_{V}} } \\
+ & {\left[2\left(D_{S^{3} C^{0}}-D_{S^{1} C^{2}}\right)\left(D_{S^{1} C^{2}}+D_{S^{1} C^{0}}\right)+D_{S^{2} C^{0}}^{2}+D_{S^{1} C^{1}}^{2}-D_{S^{2} C^{1}}^{2}\right] \sin ^{2} \theta_{C_{V}} } \\
+ & {\left[2\left(D_{S^{1} C^{2}}+D_{S^{1} C^{0}}\right) D_{S^{2} C^{0}}-2 D_{S^{2} C^{1}} D_{S^{1} C^{1}}\right] \sin \theta_{C_{V}} } \\
+ & {\left[\left(D_{S^{1} C^{2}}+D_{S^{1} C^{0}}\right)^{2}-D_{S^{1} C^{1}}^{2}\right]=0 }
\end{aligned}
$$

Note that the coefficients of equation 82 are functions of only $\tau_{o}$.

A numerical search can be performed to solve for the value of $\tau_{o}$. For a given value of $\tau_{o}$, compute all the values of $A_{C_{R}}, B_{C_{R}}, C_{C_{R}}, D_{C_{R}}, E_{C_{R}}$, and $F_{C_{R}}$ from equation 71 . Compute the values of $C_{S 0}, C_{C 0}, C_{0}$, $C_{S 1}, C_{C 1}, C_{1}$, and $C_{S 2}$ from equation 76 . Compute the values of $D_{S^{3} C^{0}}, D_{S^{2} C^{1}}, D_{S^{1} C^{2}}, D_{S^{2} C^{0}}, D_{S^{1} C^{1}}$, and $D_{S^{1} C^{0}}$ from equation 78 . The roots of the quartic defined in equation 82 can now be computed to give four possible values of $\sin \theta_{C_{V}}$. Using the inverse sine function, it is possible to find four possible values of $\theta_{C_{V}}$ between $-\pi / 2$ and $\pi / 2$. However, it is possible for the proper value of $\theta_{C_{V}}$ to fall between $-\pi$ and $\pi$. Thus, from the four possible values of $\sin \theta_{C_{V}}$, there are eight possible values of $\theta_{C_{V}}$. The proper values of $\theta_{C_{V}}$ can be resolved by checking each of the eight possible values in equation 80 . This will result in four possible values of $\theta_{C_{V}}$ between $-\pi$ and $\pi$. Equation 72 can be used to compute the possible values of $\tan \theta_{C_{R}}$. As in the previous section, equation 54 can be used to find the absolute value of $\cos \theta_{C_{R}}$ :

$$
\left|\cos \theta_{C_{R}}\right|=\frac{1}{\sqrt{1+\tan ^{2} \theta_{C_{R}}}}
$$

The possible values of $\rho$ are calculated from equation 69. The proper sign of $\cos \theta_{C_{R}}$ is selected using the fact that $\rho$ must be positive. With the values of $\tan \theta_{C_{R}}$ and $\cos \theta_{C_{R}}$, the values of $\theta_{C_{R}}$ can be found. The proper value of $C_{V}$ can be computed from equation 65 . There is now enough information to calculate four possible values for $\vec{C}_{R}$ and $\vec{C}_{V}$. The values of the integration constants $P, G$, and $K$ can be computed from equations 33. The $\tilde{V}$ and $\tilde{R}$ vectors can be computed from equations 27. Given the initial position, initial velocity, and gravity, the values of the final position and velocity can be computed from equation 26 . The problem then reduces to finding the values of $\tau_{o}$ that meet the desired final position and velocity. It will be shown in section XI that the value of $\tau_{o}$ can be bounded analytically. Thus, the problem is reduced to a one-dimensional bounded search.

\section{Bounding the Optimal Control Problem}

\section{XI.A. Optimal Control Problem Lower Boundary}

A lower boundary on the performance index of the problem can be found by solving the problem with no final position constraint. In this case, the optimal control is a constant thrust direction with a thrust acceleration which can be off its lower and upper boundaries. This subproblem can be solved analytically. Keeping the final velocity constraint and dropping the final position constraint from the problem can be achieved by choosing $\vec{\sigma}_{R}=0$. This results in the following condition:

$$
\begin{aligned}
\vec{C}_{R}=0 \quad \text { (Inactive final position constraint) } \\
\vec{\lambda}_{V}=\vec{C}_{V}=\text { constant } \quad \text { (Inactive final position constraint) }
\end{aligned}
$$

From equations 14, it can be seen that $E=F=0$. From equation 15, the optimal unit thrust direction reduces to

$$
\hat{u}=-\frac{\vec{\lambda}_{V}}{\left|\vec{\lambda}_{V}\right|}=-\frac{\vec{C}_{V}}{\sqrt{D}}
$$

The unit thrust direction is constant for the entire optimal trajectory. The thrust acceleration magnitude switching structure (SS) was defined in equation 18. Since both $\vec{\lambda}_{V}$ and $\hat{u}$ are constant, then the SS is constant over the entire optimal trajectory. Thus, the thrust acceleration magnitude will be either on its 
upper boundary, lower boundary, or off-boundary for the entire optimal trajectory. Since the unit thrust vector is constant, equation 26 can be written as:

$$
\begin{aligned}
\left(\vec{V}_{f}-\vec{V}_{o}\right)-\vec{g} \tau_{o} & =\left[\int_{0}^{\tau_{o}} a_{T} d \tau\right] \hat{u} \\
\left(\vec{R}_{f}-\vec{R}_{o}\right)-\vec{V}_{o} \tau_{o}-\frac{1}{2} \vec{g} \tau_{o}^{2} & =\left[\int_{0}^{\tau_{o}} \int_{0}^{\tau_{o}} a_{T} d \tau d \tau\right] \hat{u}
\end{aligned}
$$

The integrated equation for velocity can be used to determine the constant unit thrust acceleration direction required to meet the final velocity constraint as

$$
\hat{u}=\frac{\left(\vec{V}_{f}-\vec{V}_{o}\right)-\vec{g} \tau_{o}}{\left[\int_{0}^{\tau_{o}} a_{T} d \tau\right]}
$$

This shows that the optimal unit thrust direction is parallel to $\tilde{V}$. The unit thrust vector must have length of one. Taking the vector dot product of equation 88 with itself and setting it equal to one results in

$$
\left[\int_{0}^{\tau_{o}} a_{T} d \tau\right]^{2}=[(\vec{g} \cdot \vec{g})] \tau_{o}^{2}-2\left[\left(\vec{V}_{f}-\vec{V}_{o}\right) \cdot \vec{g}\right] \tau_{o}+\left[\left(\vec{V}_{f}-\vec{V}_{o}\right) \cdot\left(\vec{V}_{f}-\vec{V}_{o}\right)\right]
$$

Equation 89 gives the value of the performance index, the integral of the thrust acceleration, as a function of the time-of-flight. The average value of the thrust acceleration is given as

$$
a_{T_{a v g}}=\frac{\int_{0}^{\tau_{o}} a_{T} d \tau}{\tau_{o}}=\frac{\sqrt{[(\vec{g} \cdot \vec{g})] \tau_{o}^{2}-2\left[\left(\vec{V}_{f}-\vec{V}_{o}\right) \cdot \vec{g}\right] \tau_{o}+\left[\left(\vec{V}_{f}-\vec{V}_{o}\right) \cdot\left(\vec{V}_{f}-\vec{V}_{o}\right)\right]}}{\tau_{o}}
$$

Since the thrust acceleration can vary between $a_{T_{\min }}$ and $a_{T_{\max }}$, then the average value of thrust acceleration over any time interval is also bounded by the maximum and minimum acceleration values.

Consider the case when the thrust acceleration is off-boundary. An equation for the time-of-flight that minimizes the unbounded performance index can be written by taking the derivative of equation 89 with respect to $\tau_{o}$ and setting it equal to zero. This is referred to as the "unbounded performance index" because it does not account for the thrust acceleration bounds, hence

$$
\tau_{\text {ounbounded }}=\frac{\left[\left(\vec{V}_{f}-\vec{V}_{o}\right) \cdot \vec{g}\right]}{(\vec{g} \cdot \vec{g})}
$$

It is simple to verify that the unbounded time-of-flight will cause the unit thrust direction to be perpendicular to the gravitational vector, as shown in equation 22. It will also make $\mathrm{SS}=1$. Substitution of equation 91 into equation 89 results in the unbounded optimal performance index as

$$
\left[\int_{0}^{\tau_{o}} a_{T} d \tau\right]_{\text {unbounded }}=\sqrt{\left[\left(\vec{V}_{f}-\vec{V}_{o}\right) \cdot\left(\vec{V}_{f}-\vec{V}_{o}\right)\right]-\frac{\left[\left(\vec{V}_{f}-\vec{V}_{o}\right) \cdot \vec{g}\right]^{2}}{(\vec{g} \cdot \vec{g})}}
$$

The average thrust acceleration necessary to achieve this performance index value can be found by substitution of equation 91 into equation 90 and use of the trigonometric identity in equation 54 .

$$
a_{T_{\text {avg }} \text { unbounded }}=|\vec{g}| \tan \theta_{\Delta V g}
$$

where $\theta_{\Delta V g}$ is the angle between the gravity vector, $\vec{g}$, and the vector representing the change in velocity from the initial to the final state, $\left(\vec{V}_{f}-\vec{V}_{o}\right)$. Note that this angle is bounded such that $0^{\circ} \leq \theta_{\Delta V g} \leq 180^{\circ}$.

Recall that in this special case, the thrust acceleration is applied perpendicular to the gravity vector. When $\tan \theta_{\Delta V g}<0$, this implies that the problem is not controllable when thrust is applied only perpendicular to the gravity vector. When this occurs, the thrust acceleration magnitude must be on the maximum or minimum boundary. 
For the case when $90^{\circ} \leq \theta_{\Delta V g} \leq 180^{\circ}$, the change in velocity requires the thrust to act against gravity. Thus, a component of the acceleration must be in the direction parallel to gravity. In order to minimize the gravity losses, the minimum time solution is sought. This means that the optimal solution uses the maximum acceleration possible.

For the case when $0^{\circ} \leq \theta_{\Delta V g}<90^{\circ}$, the change in velocity allows some use of gravity to achieve it. The optimal component of acceleration in the channel parallel to gravity is zero. In this case, gravity is allowed to carry the vehicle along the gravity channel while all control effort is focused on the velocity perpendicular to the gravity vector. However, it is possible that this "unbounded" optimal solution results in an average acceleration which is outside the acceleration limits. In this case, the acceleration limit closest to the average acceleration is the bounded optimal solution. From equation ??, a quadratic equation for the time-of-flight when the thrust acceleration is on one of its boundaries can be written

$$
\left[(\vec{g} \cdot \vec{g})-a_{T_{l i m i t}}^{2}\right] \tau_{o}^{2}-2\left[\left(\vec{V}_{f}-\vec{V}_{o}\right) \cdot \vec{g}\right] \tau_{o}+\left[\left(\vec{V}_{f}-\vec{V}_{o}\right) \cdot\left(\vec{V}_{f}-\vec{V}_{o}\right)\right]=0
$$

Only positive roots of this equation are possible solutions. The value of the performance index is then given by the value of $a_{T_{l i m i t}}$ multiplied by the positive roots of this equation. For real world problems, only real solutions of this equation are allowed. By examining the discriminant of this quadratic equation, a necessary condition on $a_{T_{l i m i t}}$ for real solutions is found to be: ${ }^{24}$

$$
a_{T_{\text {limit }}}^{2} \geq|\vec{g}|^{2} \sin ^{2} \theta_{\Delta V g}
$$

Thus the physics of the problem impose a lower limit on the possible thrust acceleration magnitude. For a solution to this problem, the maximum thrust acceleration limit must be greater than this value.

There is now enough information to find the solution to this subproblem. The equations derived in this section can be used to evaluate the performance index for the cases when $a_{T}=a_{T_{\min }}, a_{T}=a_{T_{\max }}$, and $a_{T_{\min }} \leq a_{T} \leq a_{T_{\max }}$. The solution which gives the minimum value of the performance index while satisfying the constraints is a lower bound of the problem.

\section{XI.B. Optimal Control Problem Upper Boundary}

Any feasible solution to the problem is an upper bound on the performance index. Consider a trajectory that is comprised of two segments with constant thrust acceleration magnitude and direction. It is desired to find the thrust magnitude, thrust direction, and segment times that will take a vehicle from an initial position and velocity state to a final position and velocity state.

The equations of motion from equation 1 can be easily integrated along each segment, due to the constant thrust acceleration and gravity. The first segment runs from the initial state and time to the unknown switching state and time. The second segment runs from the unknown switching state and time to the final state and time. Combining the two equations to eliminate the unknown switching state and time results in

$$
\begin{aligned}
\tilde{V} & =\left[a_{T_{2}} \tau_{2}\right] \hat{u}_{2}+\left[a_{T_{1}} \tau_{1}\right] \hat{u}_{1} \\
\tilde{R} & =\left[\frac{1}{2} a_{T_{2}} \tau_{2}^{2}\right] \hat{u}_{2}+\left[\frac{1}{2} a_{T_{1}}\left(\tau_{2}+\tau\right) \tau_{1}\right] \hat{u}_{1}
\end{aligned}
$$

where

$$
\begin{aligned}
\tau & =\left(t_{f}-t_{o}\right)=\text { Total time-of-flight } \\
\tau_{1} & =\left(t_{s}-t_{o}\right)=\text { Time-of-flight for segment } 1 \\
\tau_{2} & =\left(t_{f}-t_{s}\right)=\text { Time-of-flight for segment } 2
\end{aligned}
$$

Note that

$$
\tau=\tau_{1}+\tau_{2}
$$

Define the ratio of $\tau_{2}$ to $\tau$ as

$$
\rho=\frac{\tau_{2}}{\tau}
$$

Note that since $0 \leq \tau_{2} \leq \tau$, then it must be true that $0 \leq \rho \leq 1$. Now $\tau_{1}$ and $\tau_{2}$ can be written in terms of $\tau$ and $\rho$ as

$$
\begin{aligned}
\tau_{1} & =\tau(1-\rho) \\
\tau_{2} & =\tau \rho
\end{aligned}
$$


Substitute equations 99 into equations 95 to obtain

$$
\begin{aligned}
\frac{\tilde{V}}{\tau} & =\left[a_{T_{2}} \rho\right] \hat{u}_{2}+\left[a_{T_{1}}(1-\rho)\right] \hat{u}_{1} \\
\frac{\tilde{R}}{\tau^{2}} & =\left[\frac{1}{2} a_{T_{2}} \rho^{2}\right] \hat{u}_{2}+\left[\frac{1}{2} a_{T_{1}}\left(1-\rho^{2}\right)\right] \hat{u}_{1}
\end{aligned}
$$

Solving these equations for the thrust direction unit vectors yields

$$
\begin{aligned}
& \hat{u}_{1}=\frac{[2 \tilde{R}-\tilde{V} \tau \rho]}{a_{T_{1}} \tau^{2}(1-\rho)} \\
& \hat{u}_{2}=\frac{[-2 \tilde{R}+\tilde{V} \tau(1+\rho)]}{a_{T_{2}} \tau^{2} \rho}
\end{aligned}
$$

Note that the thrust direction vectors must be unit vectors by definition. Taking the vector dot product of $\hat{u}_{1}$ and $\hat{u}_{2}$ from equation 101 with themselves, setting them equal to one, and rearranging results in

$$
\begin{aligned}
& a_{T_{1}}^{2}=\frac{\left[4(\tilde{R} \cdot \tilde{R})-4(\tilde{R} \cdot \tilde{V}) \tau \rho+(\tilde{V} \cdot \tilde{V}) \tau^{2} \rho^{2}\right]}{\tau^{4}(1-\rho)^{2}} \\
& a_{T_{2}}^{2}=\frac{\left[4(\tilde{R} \cdot \tilde{R})-4(\tilde{R} \cdot \tilde{V}) \tau(1+\rho)+(\tilde{V} \cdot \tilde{V}) \tau^{2}(1+\rho)^{2}\right]}{\tau^{4} \rho^{2}}
\end{aligned}
$$

For any given value of $\tau$, it will be useful to know the value of $\rho$ which makes the thrust acceleration of each segment equal to a given value (for example, the maximum or minimum acceleration limits). Rearranging equations 102 results in quadratic equations for $\rho$ as a function of $a_{T}$ and $\tau$. For $a_{T_{1}}$, we have

$$
\begin{aligned}
& {\left[-a_{T_{1}}^{2} \tau^{4}+(\tilde{V} \cdot \tilde{V}) \tau^{2}\right] \rho^{2} } \\
+ & {\left[2 a_{T_{1}}^{2} \tau^{4}-4(\tilde{R} \cdot \tilde{V}) \tau\right] \rho } \\
+ & {\left[-a_{T_{1}}^{2} \tau^{4}+4(\tilde{R} \cdot \tilde{R})\right]=0 }
\end{aligned}
$$

For $a_{T_{2}}$, we have

$$
\begin{aligned}
& {\left[-a_{T_{2}}^{2} \tau^{4}+(\tilde{V} \cdot \tilde{V}) \tau^{2}\right] \rho^{2} } \\
+ & {\left[2(\tilde{V} \cdot \tilde{V}) \tau^{2}-4(\tilde{R} \cdot \tilde{V}) \tau\right] \rho } \\
+ & {\left[(\tilde{V} \cdot \tilde{V}) \tau^{2}-4(\tilde{R} \cdot \tilde{V}) \tau+4(\tilde{R} \cdot \tilde{R})\right]=0 }
\end{aligned}
$$

Two quadratic equations

$$
\begin{aligned}
a_{2} x^{2}+a_{1} x+a_{o} & =0 \\
b_{2} x^{2}+b_{1} x+b_{o} & =0
\end{aligned}
$$

can be solved by the same value of $x$ only when the following condition holds: ${ }^{24}$

$$
0=\left[a_{2} b_{1}-a_{1} b_{2}\right]\left[a_{1} b_{o}-a_{o} b_{1}\right]-\left[a_{2} b_{o}-a_{o} b_{2}\right]^{2}
$$

This condition can be used with equations 103 and 104 to find a polynomial equation for $\tau$ as a function of the initial state, final state, gravity, and thrust acceleration magnitude on each segment. The following 
condition is obtained:

$$
\begin{aligned}
& 0=\left\{\left[(\tilde{V} \cdot \tilde{V}) \tau^{2}-a_{T_{1}}^{2} \tau^{4}\right]\left[-4(\tilde{R} \cdot \tilde{V}) \tau+2(\tilde{V} \cdot \tilde{V}) \tau^{2}\right] \ldots\right. \\
& \left.-\left[-4(\tilde{R} \cdot \tilde{V}) \tau+2 a_{T_{1}}^{2} \tau^{4}\right]\left[(\tilde{V} \cdot \tilde{V}) \tau^{2}-a_{T_{2}}^{2} \tau^{4}\right] \quad\right\} \ldots \\
& \text { * }\left\{\left[-4(\tilde{R} \cdot \tilde{V}) \tau+2 a_{T_{1}}^{2} \tau^{4}\right]\left[(\tilde{V} \cdot \tilde{V}) \tau^{2}-4(\tilde{R} \cdot \tilde{V}) \tau+4(\tilde{R} \cdot \tilde{R})\right] \ldots\right. \\
& \left.-\left[4(\tilde{R} \cdot \tilde{R})-a_{T_{1}}^{2} \tau^{4}\right]\left[-4(\tilde{R} \cdot \tilde{V}) \tau+2(\tilde{V} \cdot \tilde{V}) \tau^{2}\right] \quad\right\} \cdots \\
& -\left\{\left[(\tilde{V} \cdot \tilde{V}) \tau^{2}-a_{T_{1}}^{2} \tau^{4}\right]\left[(\tilde{V} \cdot \tilde{V}) \tau^{2}-4(\tilde{R} \cdot \tilde{V}) \tau+4(\tilde{R} \cdot \tilde{R})\right] \ldots\right. \\
& \left.-\left[4(\tilde{R} \cdot \tilde{R})-a_{T_{1}}^{2} \tau^{4}\right]\left[(\tilde{V} \cdot \tilde{V}) \tau^{2}-a_{T_{2}}^{2} \tau^{4}\right] \quad\right\}^{2}
\end{aligned}
$$

Each coefficient of the polynomial is a function only of the initial state, final state, gravity, and the thrust acceleration magnitude on each segment. An initial attempt to collect terms of $\tau$ by hand was abandoned. Instead, a Matlab script was written to symbolically convolve each subpolynomial in tau in order to define the coefficients. The resultant 10th order polynomial in $\tau$ is much too large to put into print.

There is now enough information to solve the problem for a given initial state, final state, gravity, and maximum and minimum thrust acceleration. The coefficients of equation 107 are evaluated, assuming thrust acceleration magnitudes for each segment. All positive, real roots of this equation are potential solutions for $\tau$. If no positive, real roots exist, different thrust acceleration magnitudes are chosen. Once potential values of $\tau$ are available, the roots of either equations 103 or 104 are found. The roots that are between zero and one are potential values of $\rho$. The value of the performance index is given by:

$$
\int_{0}^{\tau_{o}} a_{T} d \tau=a_{T_{1}} \tau_{1}+a_{T_{2}} \tau_{2}=\left[a_{T_{1}}(1-\rho)+a_{T_{2}} \rho\right] \tau
$$

If multiple solutions exists, the one with the lowest performance index is chosen. The unit thrust direction for each segment can then be computed from equations 101.

\section{Numerical Solution of Reduced Problem}

An algorithm to solve the optimal control problem with a constant thrust acceleration magnitude was developed. This algorithm is a mechanization of the equations from section X. Lower and upper bounds on the time-of-flight are given in section XI. The algorithm is a one-dimensional bounded search developed specifically for the problem at hand.

In order to test the proposed guidance algorithm in various scenarios, a simulation was developed in Matlab. ${ }^{43}$ The simulation models a vehicle as a point mass over a rotating, spherical planet with an inverse gravitational field. Both the navigation and control systems are assumed to be perfect. The guidance is called at a specified rate, and the solution is assumed to be instantaneously available. The vehicle rocket engine is assumed to be perfectly expanded and throttleable. In practice, the guidance commands a thrust acceleration level. Both nominal runs and monte carlo runs are completed. Each monte carlo run includes 100 dispersed trajectories. For details on the models of the simulation, see reference 24 .

A numerical optimizer was used to test the optimality of the solution from the simulation. The numerical optimization uses the Legendre pseudospectral collocation method. A pseudospectral differentiation matrix is used to write the equations of motion as a set of nonlinear algebraic equations. The problem is then written numerically as a parameter optimization problem, where the states, controls, and time-of-flight are manipulated by a numerical optimizer to achieve an optimal solution. Details of the optimization method can be found in reference 44 .

\section{XII.A. Test Case Definition}

The lander begins in a circular orbit with an altitude of 100 kilometers and an inclination of 60 degrees. It is at its maximum southern latitude when a deorbit burn is completed to put the lander into an elliptical transfer orbit with an apolune altitude of $100 \mathrm{~km}$ and a perilune altitude of $20 \mathrm{~km}$. When the lander reaches the perilune of the transfer orbit, the descent phase begins with a braking burn aligned opposite 
the planet relative velocity vector. This portion of flight is not modeled in the simulation. When the altitude drops below a threshold of $6 \mathrm{~km}$, the closed-loop guidance commands the thrust acceleration vector to achieve a desired final position and velocity state. Guidance is called every 10 seconds to generate new thrust commands. Between guidance calls, the thrust acceleration command profile from the last guidance call is followed open loop. In order to simulate a possible hazard avoidance maneuver, the target may be redesignated at a total range-to-target of 2 kilometers. If this occurs, the new landing target is loaded into guidance at the time of redesignation; the guidance has no apriori knowledge of the new landing site. When the time-to-go of the optimal flight phase drops below 10 seconds, guidance is no longer called, and the final guidance commands are followed open loop. At the termination of the optimal flight, the vehicle is over the landing site with a very small negative altitude rate and no horizontal velocity. The pitch up and vertical landing phases are not modeled in the simulation.

The guidance algorithm computes commands in a topocentric guidance frame. For this frame, the X-axis is East, the Y-axis is North, and the Z-axis is Up. The guidance algorithm assumes that all gravitational acceleration is directed along the negative Z-guidance axis. During each call to guidance, a corrected gravity term is added to account for the centripetal acceleration of the topocentric frame.

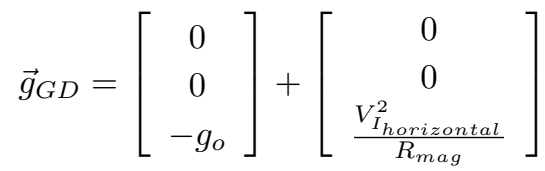

where

$$
\begin{aligned}
g_{o} & =\text { Gravitational acceleration magnitude at surface of planet } \\
V_{\text {Ihorizontal }} & =\text { Inertial velocity in the local horizontal plane } \\
R_{\text {mag }} & =\text { Radial position magnitude }
\end{aligned}
$$

The initial state of the lander is given in Table 1. The selected landing targets are given in Table 2. The lander vehicle properties are given in Table 3. The properties of the moon are given in Table 4 . The monte carlo dispersions are given in Table 5 .

\begin{tabular}{|l|l|c|}
\hline Variable & Units & Initial State \\
\hline \hline Range North of Target & $\mathrm{m}$ & 3100 \\
\hline Range East of Target & $\mathrm{m}$ & -21120 \\
\hline Geocentric Altitude & $\mathrm{m}$ & 6000 \\
\hline Planet Relative Velocity Magnitude & $\mathrm{m} / \mathrm{s}$ & 350 \\
\hline $\begin{array}{l}\text { Planet Relative Topocentric } \\
\text { Flight Path Angle }\end{array}$ & $\mathrm{deg}$ & -18.65 \\
\hline $\begin{array}{l}\text { Planet Relative Topocentric } \\
\text { Azimuth Angle }\end{array}$ & $\mathrm{deg}$ & 104 \\
\hline
\end{tabular}

Table 1. Lunar Test Case Initial State 


\begin{tabular}{|l|l|c|c|}
\hline \multirow{2}{*}{ Variable } & \multirow{2}{*}{ Units } & \multicolumn{2}{|c|}{ Landing Site } \\
\cline { 3 - 4 } & & Primary & Divert \\
\hline \hline Geocentric Latitude & $\operatorname{deg}$ & 58.9 & 58.91649 \\
\hline Longitude & $\mathrm{deg}$ & 146.73 & 146.76298 \\
\hline Geocentric Altitude & $\mathrm{m}$ & 100 & 100 \\
\hline Geocentric Altitude Rate & $\mathrm{m} / \mathrm{s}$ & -5 & -5 \\
\hline $\begin{array}{l}\text { Planet Relative } \\
\text { Horizontal Velocity }\end{array}$ & $\mathrm{m} / \mathrm{s}$ & 0 & 0 \\
\hline
\end{tabular}

Note: The divert landing site is $0.5 \mathrm{~km}$ north and $1.0 \mathrm{~km}$ east of the primary landing site.

Table 2. Lunar Test Case Landing Targets

\begin{tabular}{|l|l|c|}
\hline Variable & Units & Value \\
\hline Thrust Acceleration Magnitude & $\mathrm{m} / \mathrm{s}^{2}$ & 5.5 \\
\hline $\begin{array}{l}\text { Gravitational Acceleration Magnitude } \\
\text { at Surface of Planet }\end{array}$ & $\mathrm{m} / \mathrm{s}^{2}$ & 1.635 \\
\hline
\end{tabular}

Table 3. Lunar Test Case Vehicle Properties

\begin{tabular}{|l|l|c|}
\hline Variable & Units & Value \\
\hline \hline Equatorial Radius & $\mathrm{km}$ & 1737.4 \\
\hline Gravitational Constant & $\mathrm{km}^{3} / \mathrm{s}^{2}$ & $4.9028 \times 10^{3}$ \\
\hline Sidereal Rotation Period & hours & 655.728 \\
\hline
\end{tabular}

Table 4. Planetary Properties of the Moon

\begin{tabular}{|l|l|c|c|}
\hline Variable & Units & Mean & $\begin{array}{c}\text { Uniform } \\
\text { Dispersion }\end{array}$ \\
\hline \hline Range North of Target & $\mathrm{m}$ & 3100 & \pm 500 \\
\hline Range East of Target & $\mathrm{m}$ & -21120 & \pm 500 \\
\hline Geocentric Altitude & $\mathrm{m}$ & 6000 & \pm 100 \\
\hline $\begin{array}{l}\text { Planet Relative } \\
\text { Velocity Magnitude }\end{array}$ & $\mathrm{m} / \mathrm{s}$ & 350 & \pm 5 \\
\hline $\begin{array}{l}\text { Planet Relative Topocentric } \\
\text { Flight Path Angle }\end{array}$ & $\mathrm{deg}$ & -18.65 & \pm 0.25 \\
\hline $\begin{array}{l}\text { Planet Relative Topocentric } \\
\text { Azimuth Angle }\end{array}$ & $\mathrm{deg}$ & 104.0 & \pm 0.25 \\
\hline
\end{tabular}

Table 5. Lunar Test Case Monte Carlo Dispersions 


\section{XII.B. Flight to the Primary Target}

Table 6 contains results for flight to the primary target. A comparison is given between the optimized trajectory, the nominal trajectory from the simulation, and statistical results of the 100 dispersed trajectories at the final flight time. Target accuracy for all cases is very good, and the performance index from the nominal simulation is very close to the numerically optimized performance index. The performance index shows a variation of roughly 90 meters/second due to the dispersed initial state in the monte carlo analysis.

\begin{tabular}{|l|l|c|c|c|c|c|c|}
\hline Parameter & Units & Target & Optimizer & Nominal & Min & Mean & Max \\
\hline \hline Total Range & $\mathrm{m}$ & 0 & $1.25 \mathrm{e}-1$ & $8.55 \mathrm{e}-2$ & $8.40 \mathrm{e}-5$ & $8.99 \mathrm{e}-2$ & $2.01 \mathrm{e}-1$ \\
\hline North Range & $\mathrm{m}$ & 0 & $1.78 \mathrm{e}-2$ & $-8.55 \mathrm{e}-2$ & $-1.99 \mathrm{e}-1$ & $-8.48 \mathrm{e}-2$ & $7.53 \mathrm{e}-3$ \\
\hline East Range & $\mathrm{m}$ & 0 & $-1.24 \mathrm{e}-1$ & 0.00 & $-6.34 \mathrm{e}-2$ & $1.12 \mathrm{e}-2$ & $7.32 \mathrm{e}-2$ \\
\hline $\begin{array}{l}\text { Geocentric } \\
\text { Altitude }\end{array}$ & $\mathrm{m}$ & 100 & 100.006 & 101.217 & 100.538 & 101.393 & 102.060 \\
\hline $\begin{array}{l}\text { Geocentric } \\
\text { Altitude Rate }\end{array}$ & $\mathrm{m} / \mathrm{s}$ & -5 & -5.000 & -4.844 & -4.899 & -4.833 & -4.782 \\
\hline $\begin{array}{l}\text { Planet Relative } \\
\text { Horizontal Velocity }\end{array}$ & $\mathrm{m} / \mathrm{s}$ & 0 & $1.465 \mathrm{e}-3$ & $1.26 \mathrm{e}-2$ & $3.80 \mathrm{e}-4$ & $1.123 \mathrm{e}-2$ & $1.841 \mathrm{e}-2$ \\
\hline \hline Time-of-Flight & $\mathrm{s}$ & - & 75.2567 & 75.3535 & 74.706 & 78.896 & 91.257 \\
\hline Performance Index & $\mathrm{m} / \mathrm{s}$ & - & 413.9118 & 414.4443 & 410.883 & 433.928 & 501.914 \\
\hline
\end{tabular}

Table 6. Primary Target: Comparison of Optimizer and Simulation at Final Time

Figures 1(a) through 1(h) show trajectory plots for flight to the primary target. For each plot, the solid red line shows the nominal trajectory from the simulation, the dashed cyan line shows the trajectory from the optimizer, and each solid blue line represents one dispersed trajectory. From these figures, it can be seen that the nominal trajectory matches the optimized trajectory very well. It can also be seen that each monte carlo flight hits the desired final target state.

Figure 1(a) shows the geocentric altitude versus the total range to the target. Figure 1(b) shows the time history of the planet relative velocity magnitude. Of particular interest is Figure 1(c), which shows the groundtrack in terms of geocentric latitude versus longitude. Each dispersed trajectory indeed hits the final target point, but $65 \%$ of the cases overfly the target by more than 10 meters before turning around. This can also be seen in Figure 1(d), which show the time history of the planet relative topocentric flight path angle. The variations seen in flight path angle near the end of the trajectories show the flight characteristics of a vehicle correcting for overflight. It can also be seen that the flight path angle varies slightly between the nominal and optimized trajectories near the end of flight.

Figures 1(e) and 1(f) show the thrust direction angles. Differences can be seen between the nominal and optimized trajectories which lead to a slightly better solution for the optimizer. For the monte carlo results, the variations at the end of the trajectory show vehicle response to the overflight. However, it has been verified with the numerical optimizer that the overflight trajectories are indeed optimal for flight from each particular initial dispersed state. The overflight is predicted on each call to guidance, beginning with the very first call. This is due to the fact that a constant thrust acceleration magnitude, $a_{T}$, is used for the entire trajectory. For a particular dispersed initial state, a different value for $a_{T}$ would minimize the amount of overflight. However, for the value of $a_{T}$ used in this test case, the overflight is fuel optimal for many of the dispersed trajectories. No analysis was performed to optimize the thrust acceleration magnitude for each dispersed trajectory.

Figure 1(g) shows the total angle of attack. The difference between the nominal and optimized trajectories is most pronounced in these plots. Note that the optimal total angle of attack is not zero, although it is small. Thus, a gravity turn which thrusts opposite the velocity vector is not optimal for the constraints of this test case. The same trend is seen in the monte carlo trajectories. Figure 1(h) shows the estimated time-to-go as a function of time for each trajectory. No line is given for the optimizer because it is flown off-line. As the guidance is called every 10 seconds, the time-to-go decrements nearly linearly with time for each trajectory. 


\section{XII.C. Flight to the Divert Target}

Table 6 contains results for flight to the divert target. For this scenario, the guidance is initially given the primary landing target. At a total range-to-target of two kilometers, the divert landing target defined in Table 2 is given to the guidance. The divert landing site is five hundred meters north and one kilometer east of the primary landing site. For direct comparison, the exact initial state dispersions used for the flight to the primary target are used here. A comparison is given between the nominal trajectory to the primary target, the nominal trajectory to the divert target, and statistical results of the 100 dispersed trajectories at the final flight time. Note that the target accuracy is very good. The performance index shows a variation of roughly 40 meters/second due to the dispersed initial state.

\begin{tabular}{|l|l|c|c|c|c|c|c|}
\hline Parameter & Units & Target & Nominal Primary & Nominal Divert & Min & Mean & Max \\
\hline \hline Total Range & $\mathrm{m}$ & 0 & $8.55 \mathrm{e}-2$ & $3.62 \mathrm{e}-1$ & $8.00 \mathrm{e}-3$ & $2.47 \mathrm{e}-1$ & $4.19 \mathrm{e}-1$ \\
\hline North Range & $\mathrm{m}$ & 0 & $-8.55 \mathrm{e}-2$ & $-2.58 \mathrm{e}-1$ & $-2.62 \mathrm{e}-1$ & $-1.71 \mathrm{e}-1$ & $8.00 \mathrm{e}-3$ \\
\hline East Range & $\mathrm{m}$ & 0 & 0.00 & $2.55 \mathrm{e}-1$ & 0 & $1.74 \mathrm{e}-1$ & $3.30 \mathrm{e}-1$ \\
\hline $\begin{array}{l}\text { Geocentric } \\
\text { Altitude }\end{array}$ & $\mathrm{m}$ & 100 & 101.217 & 101.730 & 100.540 & 101.388 & 101.854 \\
\hline $\begin{array}{l}\text { Geocentric } \\
\text { Altitude Rate }\end{array}$ & $\mathrm{m} / \mathrm{s}$ & -5 & -4.844 & -4.818 & -4.894 & -4.837 & -4.812 \\
\hline $\begin{array}{l}\text { Planet Relative } \\
\text { Horizontal Velocity }\end{array}$ & $\mathrm{m} / \mathrm{s}$ & 0 & $1.26 \mathrm{e}-2$ & $4.098 \mathrm{e}-2$ & $1.894 \mathrm{e}-3$ & $2.864 \mathrm{e}-2$ & $4.232 \mathrm{e}-2$ \\
\hline \hline Time-of-Flight & $\mathrm{s}$ & - & 75.3535 & 78.675 & 77.543 & 78.555 & 84.546 \\
\hline Performance Index & $\mathrm{m} / \mathrm{s}$ & - & 414.4443 & 432.7125 & 426.487 & 432.053 & 465.003 \\
\hline
\end{tabular}

Table 7. Divert Target: Comparison of Divert and Primary Trajectories at Final Time

Figures 1(i) through 1(p) show data for the Lunar Test Case nominal flight to the primary and divert landing sites. For each plot, the solid red line shows the nominal trajectory to the divert target, the dashed cyan line shows the nominal trajectory to the primary target, and each solid blue line represents one dispersed trajectory. The two nominal trajectories are exactly the same until the total range-to-target reaches a value of two kilometers. Figure 1(i) shows the geocentric altitude versus the total range to the target. The discontinuity in the range occurs when the divert target is loaded into guidance. Figure $1(\mathrm{j})$ shows the time history of the planet relative velocity magnitude. Figure 1(c) shows the groundtrack in terms of geocentric latitude versus longitude. Note that none of the trajectories overfly the divert target. Figure 1(l) shows the time history of the planet relative topocentric flight path angle. From these plots, it can be seen that each trajectory hits the desired final target state.

Figures $1(\mathrm{~m})$ and $1(\mathrm{n})$ show the thrust direction angles. The guidance response to the divert target can be seen clearly. Especially of interest is Figure 1(o), which shows the total angle of attack. Flight to the primary target has a small total angle of attack. This shows that the optimal thrust direction is very nearly opposite the velocity vector. However, for the divert trajectory, it can be seen that thrusting along the velocity vector is not desired. For both the nominal and monte carlo flights, it can be seen that the optimal thrust profile is not aligned opposite the velocity vector during the divert maneuver. The estimated time-to-go versus time is shown in Figure 1(p). A discontinuity occurs when the divert target is loaded into guidance. Note that except for this discontinuity, the estimated time-to-go is nearly linear with time.

\section{Conclusions}

Powered descent is a problem often characterized by a desire to safely achieve a specified landing target while minimizing the amount of fuel used in the maneuver. Integration of the equations of motion with the well known fuel optimal bilinear tangent steering law results in a system of five nonlinear equations in five unknowns. It was observed that the optimal unit thrust vector lies in a plane completely defined by the initial position, initial velocity, final position, final velocity, gravity, and the time-of-flight. The major contribution of this research is the use of this Thrust Plane to reduce the dimensionality of the nonlinear 
problem. The problem with lower and upper thrust acceleration bounds can be reduced to a system of two equations in two unknowns: the time-of-flight and the angle of the velocity Lagrange multiplier in the Thrust Plane. The problem with a constant thrust acceleration can be reduced to one equation in one unknown: the time-of-flight. In addition, analytical lower and upper bounds on the time-of-flight have been derived. Thus, the problem is reduced to either a bounded two-dimensional search or a bounded one-dimensional search. The bounded one-dimensional search that defines the problem with constant thrust acceleration has been mechanized. It was demonstrated as a potential guidance algorithm for terminal descent to the surface of the moon. Its potential use for hazard avoidance divert maneuvers was shown. Several ares for future work have been identified:

1. The bounded one-dimensional search for the constant thrust acceleration solution was mechanized efficiently. Work was begun on a similar mechanization of the bounded two-dimensional search for the problem with bounded thrust acceleration. However, an efficient algorithm has not yet been found.

2. The reduction of dimensionality was done for problems with bounded thrust acceleration. However, some problems may be better described by a bounded thrust magnitude. The concept of the Thrust Plane may be applicable to such problems, allowing for reduction of dimensionality for bounded and constant thrust magnitude solutions.

3. During the course of this research, it was noted that the bounded thrust acceleration problem could be reduced to one variable if enough numerical precision were available to compute the coefficients of a polynomial in time-of-flight with order greater than twenty. The coefficients themselves are high order polynomials of the parameters of the problem, and thus are prone to numerical error. This avenue was not investigated in the current research.

4. The derivation of the optimal control law does not constrain the trajectory to fly above the surface of the planet. The trajectory is determined by the initial state, final state, and gravity. It is very possible that the optimal trajectory will fly subsurface. For the test cases of this research, the initial states were chosen to avoid subsurface flight. This is not difficult, but care must be taken to choose a proper initial state such that the trajectory will fly above the surface for all dispersed states. Future work may be done to include a constraint on altitude to ensure flight above the surface.

5. For guidance implementation of the constant thrust acceleration algorithm, it may be desired to search for the best value of the constant thrust acceleration. This may allow for lower fuel usage for dispersed trajectories. It may also be possible to select proper thrust acceleration magnitudes that avoid subsurface flight.

6. The algorithm was tested in a three degree-of-freedom simulation with perfect control and perfect navigation. While this is a good environment for proof-of-concept work, the algorithm should be tested in a high-fidelity, six degree-of-freedom environment.

\section{Acknowledgments}

This research was performed at The University of Texas at Austin and the National Aeronautics and Space Administration Johnson Space Center under a Johnson Space Center Fellowship.

\section{References}

1 "NASA - Constellation Main," 2009, http://www.nasa.gov/mission_pages/ constellation/main/index.html.

2 "Google Lunar X Prize," 2009, http://www.googlelunarxprize.org/.

3 "China launches first Moon orbiter," Oct. 2007, http://news.bbc.co.uk/ 2/hi/asia-pacific/7059356.stm.

${ }^{4}$ Matsumotoa, K., Kamimoria, N., Takizawab, Y., Katob, M., Odaa, M., SachikoWakabayashia, Kawamotoa, S., Okadab, T., Iwatab, T., and Ohtakeb, M., "Japanese Lunar Exploration Long-Term Plan," Acta Astronautica, Vol. 59, 2006 , pp. 68-76.

${ }^{5}$ Sostaric, R., "Lunar Descent Reference Trajectory, Version 2.0," Tech. rep.

${ }^{6}$ Sostaric, R. R. and Rea, J. R., "Powered Descent Guidance Methods for the Moon and Mars," AIAA Guidance, Navigation, and Control Conference and Exhibit, American Institute of Aeronautics and Astronautics, Inc., San Francisco, CA, Aug. 2005, AIAA-2005-6287.

${ }^{7}$ Ploen, S. R., Acikmese, A. B., and Wolf, A., "A Comparison of Powered Descent Guidance Laws for Mars Pinpoint Landing," AIAA/AAS Astrodynamics Specialist Conference and Exhibit, American Institute of Aeronautics and Astronautics, Inc., Keyston, CO, Aug. 2006, AIAA-2006-6676. 
${ }^{8}$ Klumpp, A. R., “Apollo Lunar Descent Guidance," Tech. Rep. R-695, MIT Charles Stark Draper Laboratory, Cambridge, MA, 1971.

${ }^{9}$ Klumpp, A. R., "Apollo Lunar Descent Guidance," Automatica, Vol. 10, March 1974, pp. 133-146.

${ }^{10}$ Wong, E., Singh, G., and Masciarelli, J., "Guidance and Control Design for Hazard Avoidance and Safe Landing on Mars," Journal of Spacecraft and Rockets, Vol. 43, No. 2, 2006, pp. 378-384.

${ }^{11}$ McHenry, R. L., Brand, T. J., Long, A. D., Cockrell, B. F., and Thibodeau, J. R., "Space Shuttle Ascent Guidance, Navigation, and Control," The Journal of the Astronautical Sciences, Vol. 27, No. 1, January-March 1979, pp. 1-38.

${ }^{12}$ Fill, T. J., "Introduction to Bi-Linear Tangent Steering For Shuttle Ascent and Aborts," Tech. Rep. EGB-89-108, SHUTTLE-89-022, The Charles Stark Draper Laboratory, Inc., Cambridge, MA, May 1989.

${ }^{13}$ Jaggers, R. F., "Shuttle Powered Explicit Guidance (PEG) Algorithm," Tech. Rep. JSC-261-22, NASA Johnson Space Center, Houston, TX, Nov. 1992.

${ }^{14}$ Delporte, M. and Sauvinet, F., "Explicit Guidance Law for Manned Spacecraft," Aerospace Design Conference, American Institute of Aeronautics and Astronautics, Inc., Irvine, CA, Feb. 1992, AIAA-92-1145.

${ }^{15}$ D'Souza, C. N., "An Optimal Guidance Law for Planetary Landing," AIAA Guidance, Navigation, and Control Conference, American Institute of Aeronautics and Astronautics, Inc., New Orleans, LA, Aug. 1997, AIAA-1997-3709.

${ }^{16}$ Najson, F. and Mease, K. D., "Computationally Inexpensive Guidance Algorithm for Fuel-Efficient Terminal Descent," Journal of Guidance, Control, and Dynamics, Vol. 29, No. 4, July-August 2006, pp. 955-964.

${ }^{17}$ Ross, I. M., "How to Find Minimum-Fuel Controllers," AIAA Guidance, Navigation, and Control Conference and Exhibit, American Institute of Aeronautics and Astronautics, Inc., Providence, RI, Aug. 2004, pp. 1-10, AIAA-2004-5346.

${ }^{18}$ Ueno, S. and Yamaguchi, Y., "3-Dimensional Near-Minimum Fuel Guidance Law of a Lunar Landing Module," AIAA Guidance, Navigation, and Control Conference and Exhibit, American Institute of Aeronautics and Astronautics, Inc., Portland, OR, Aug. 1999, AIAA-1999-3983.

${ }^{19}$ Uchiyama, K., "Guidance Law for Lunar Lander with Input Constraint," AIAA Guidance, Navigation and Control Conference and Exhibit, American Institute of Aeronautics and Astronautics, Inc., Hilton Head, SC, Aug. 2007, AIAA-20076848.

${ }^{20}$ McInnes, C. R., "Path Shaping Guidance for Terminal Lunar Descent," Acta Astronautica, Vol. 36, No. 7, 1995, pp. 367377.

${ }^{21}$ Chomel, C., Development of an Analytical Guidance Algorithm for Lunar Descent, Ph.D. thesis, The University of Texas at Austin, Austin, TX, Dec. 2007.

${ }^{22}$ Acikmese, B. and Ploen, S. R., "Convex Programming Approach to Powered Descent Guidance for Mars Landing," Journal of Guidance, Control, and Dynamics, Vol. 30, No. 5, September-October 2007, pp. 1353-1366.

${ }^{23}$ Acikmese, B., Blackmore, L., Scharf, D. P., and Wolf, A., "Enhancements on the Convex Programming Based Powered Descent Guidance Algorithm for Mars Landing," AIAA/AAS Astrodynamics Specialist Conference and Exhibit, American Institute of Aeronautics and Astronautics, Inc., Honolulu, HI, Aug. 2008, AIAA-2008-6426.

${ }^{24}$ Rea, J., An Investigation of Fuel Optimal Terminal Descent, Ph.D. thesis, The University of Texas at Austin, Austin, TX, May 2009.

${ }^{25}$ Lawden, D. F., "Optimal Rocket Trajectories," ARS Journal, Vol. 27, No. 12, 1957, pp. 22-27.

${ }^{26}$ Lawden, D. F., Optimal Trajectories for Space Navigation, Butterworths Mathematical Texts, Butterworh, London, 1963.

${ }^{27}$ Miele, A., "Calculus of Variations in Applied Aerodynamics and Flight Mechanics," Optimization Techniques with Applications to Aerospace Systems, edited by G. Leitmann, Academic Press, New York, 1962.

${ }^{28}$ Arthur E. Bryson, J. and Ho, Y.-C., Applied Optimal Control, Hemisphere Publishing Corporation, Bristol, PA, revised printing ed., 1975.

${ }^{29}$ Marec, J. P., Optimal Space Trajectories, Vol. 1 of Studies in Astronautics, Elsevier Scientific Publishing Company, Amsterdam, 1979.

${ }^{30}$ Leitmann, G., "Class of Variational Problems in Rocket Flight," Journal of the Aero/Space Sciences, Vol. 26, No. 9, 1959 , pp. $586-591$.

${ }^{31}$ Leitmann, G., "Variational Problems with Bounded Control Variables," Optimization Techniques with Applications to Aerospace Systems, edited by G. Leitmann, Academic Press, New York, 1962.

${ }^{32}$ Topcu, U., Casoliva, J., and Mease, K. D., "Minimum-Fuel Powered Descent for Mars Pinpoint Landing," Journal of Spacecraft and Rockets, Vol. 44, No. 2, March-April 2007, pp. 324-331.

${ }^{33}$ Vinh, N. X., Optimal Trajectories in Atmospheric Flight, Elsevier Scientific Publishing Company, New York, 1981.

${ }^{34}$ Hull, D., Optimal Control Theory for Applications, Springer, New York, 2003.

${ }^{35}$ Yang, T. L., "A Targeting Scheme for Fuel Optimal Rocket Trajectories - With Applications to the LM Descent Braking Phase," Tech. Rep. NASA TM-71-2014-1, Bellcomm, Inc., Washington, D.C., Jan. 1971.

${ }^{36}$ Meditch, J., "On the Problem of Optimal Thrust Programming for a Lunar Soft Landing," IEEE Transactions on Automatic Control, Vol. 9, No. 4, 1964, pp. 477-484.

${ }^{37}$ Yang, T. L., "Optimal Control for a Rocket in a Three-Dimensional Central Force Field," Tech. Rep. NASA TM-692011-2, Bellcomm, Inc., Washington, D.C., May 1969.

${ }^{38}$ Feeley, T., Approximate Optimal Guidance for the Advanced Launch System, Ph.D. thesis, The University of Texas at Austin, Austin, TX, May 1992.

${ }^{39}$ Feeley, T. and Speyer, J., "Techniques for Developing Approximate Optimal Advanced Launch System Guidance," Journal of Guidance, Control, and Dynamics, Vol. 17, No. 5, September-October 1994, pp. 889-896.

${ }^{40}$ Leung, M. and Calise, A., "Hybrid Approach to Near-Optimal Launch Vehicle Guidance," Journal of Guidance, Control, and Dynamics, Vol. 17, No. 5, September-October 1994, pp. 881-888. 
${ }^{41}$ Hull, D., "Optimal Guidance for Lunar Ascent," Advances in the Astronautical Sciences, American Astronautical Society, Savannah, GA, Feb. 2009, AAS 09-119.

${ }^{42}$ Zwillinger, D., editor, CRC Standard Mathematical Tables and Formulae, CRC Press, Boca Raton, Florida, 30th ed., 1996.

43 "The MathWorks - MATLAB and Simulink for Technical Computing," 2009, http://www.mathworks.com.

${ }^{44}$ Ross, I., "User's manual for DIDO: A MATLAB Application Package for Solving Optimal Control Problems," Tech. rep. 

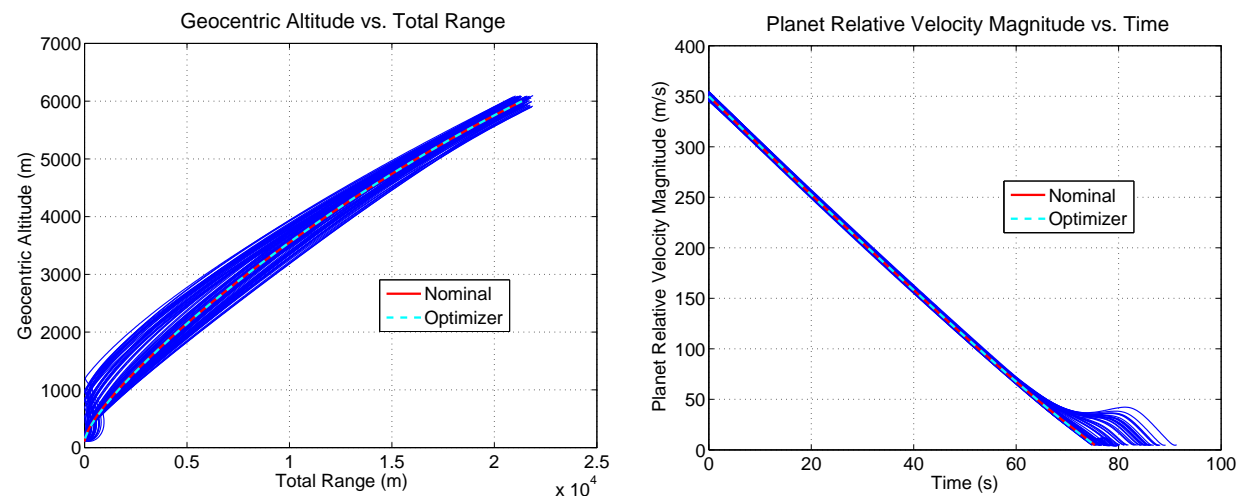

(a) Primary Target: Geocentric Altitude vs. To- (b) Primary Target: Planet Relative Velocity tal Range

Magnitude vs. Time
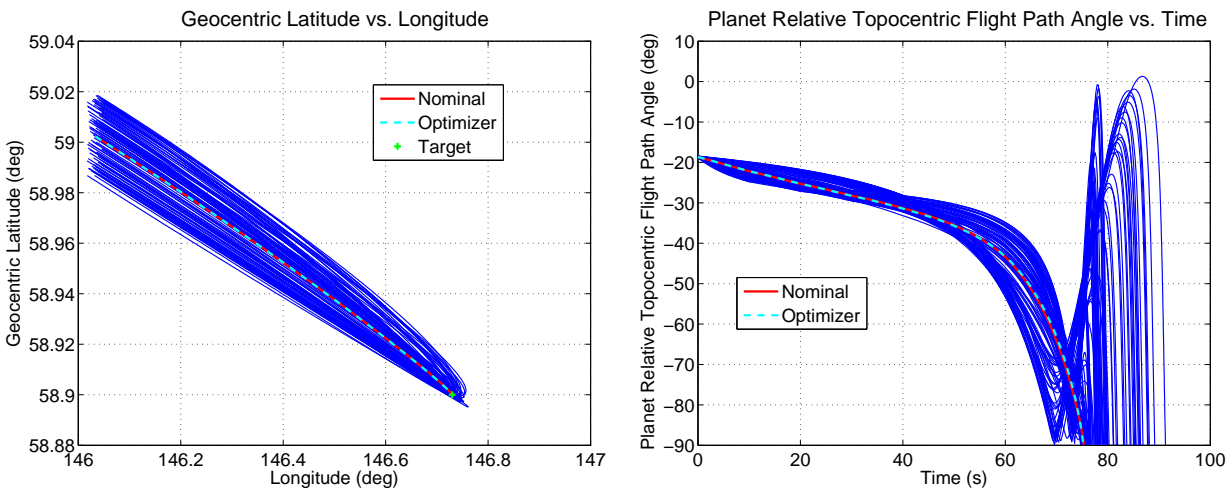

(c) Primary Target: Geocentric Latitude vs. (d) Primary Target: Planet Relative Topocentric Longitude

Flight Path Angle vs. Time
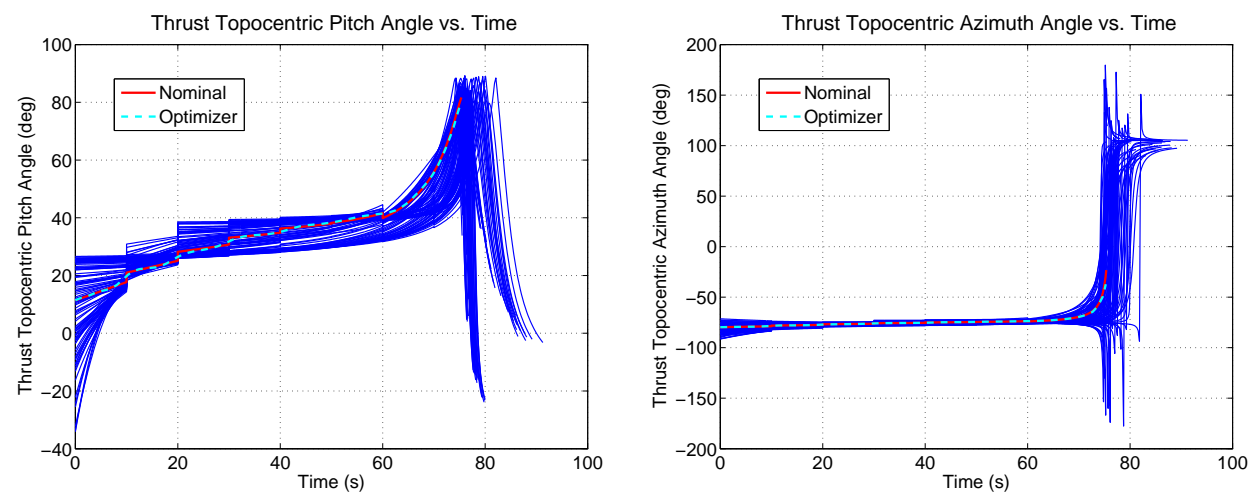

(e) Primary Target: Thrust Topocentric Pitch (f) Primary Target: Thrust Topocentric AzAngle vs. Time

imuth Angle vs. Time
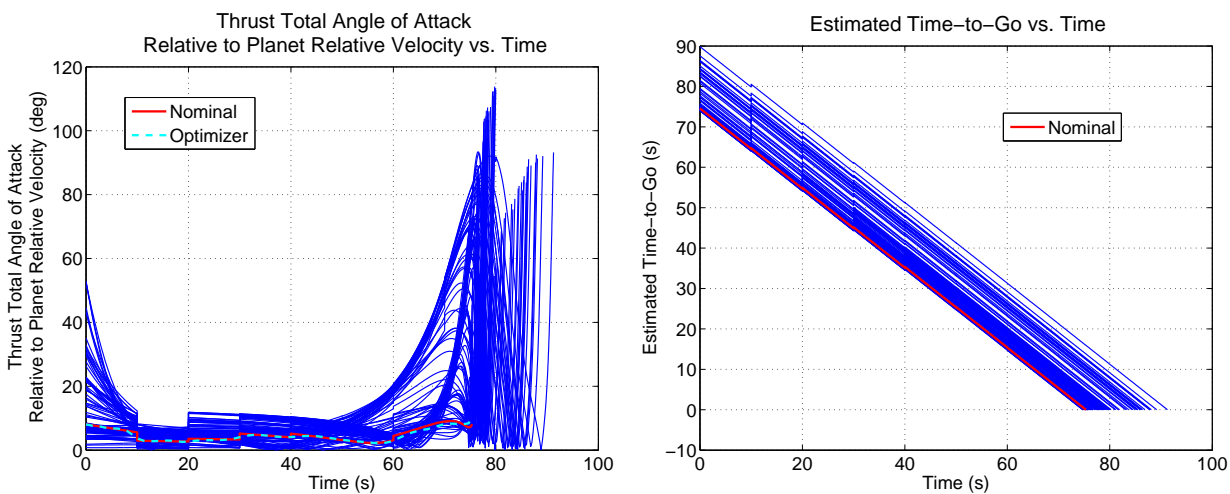

(g) Primary Target: Thrust Total Angle of At- (h) Primary Target: Estimated Time-to-Go vs. tack Relative to Planet Relative Velocity vs. Time Time 

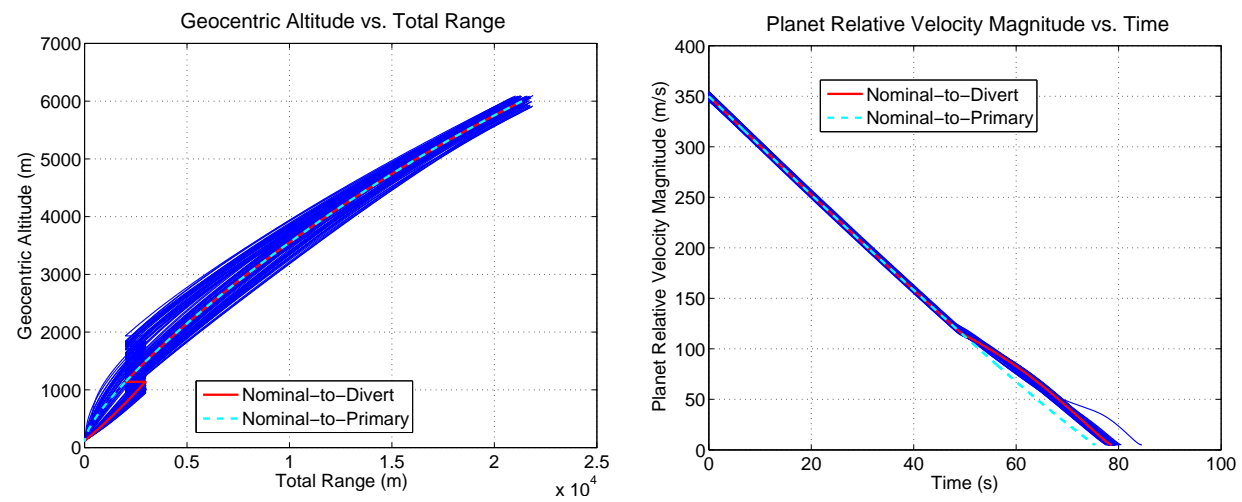

(i) Divert Target: Geocentric Altitude vs. Total (j) Divert Target: Planet Relative Velocity MagRange nitude vs. Time
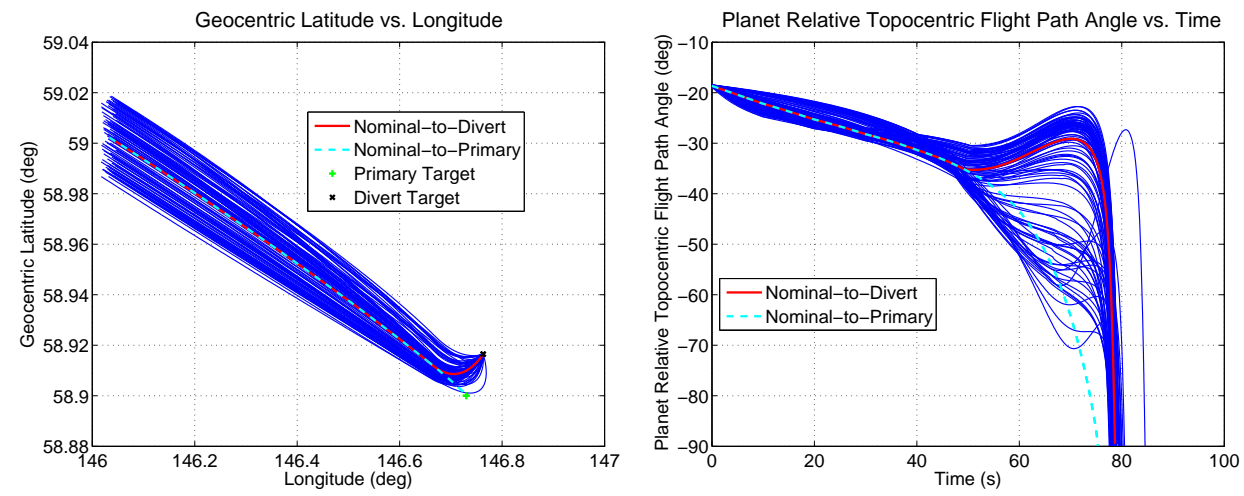

(k) Divert Target: Geocentric Latitude vs. Longitude

(l) Divert Target: Planet Relative Topocentric

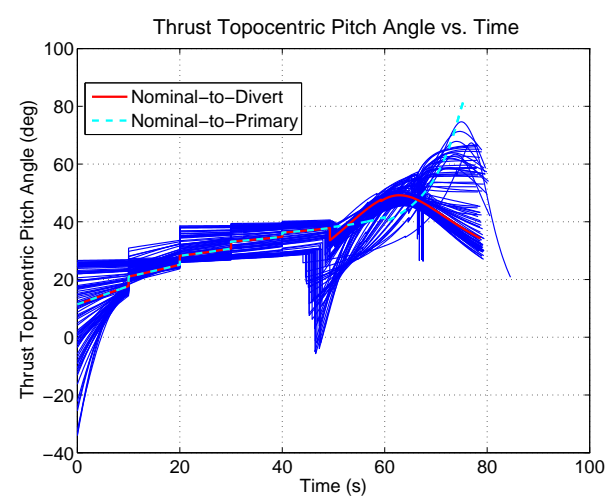

Flight Path Angle vs. Time

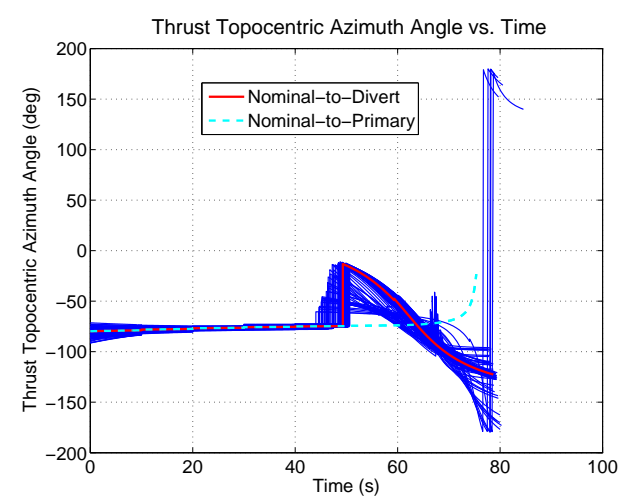

(m) Divert Target: Thrust Topocentric Pitch (n) Divert Target: Thrust Topocentric Azimuth Angle vs. Time

\section{Angle vs. Time}
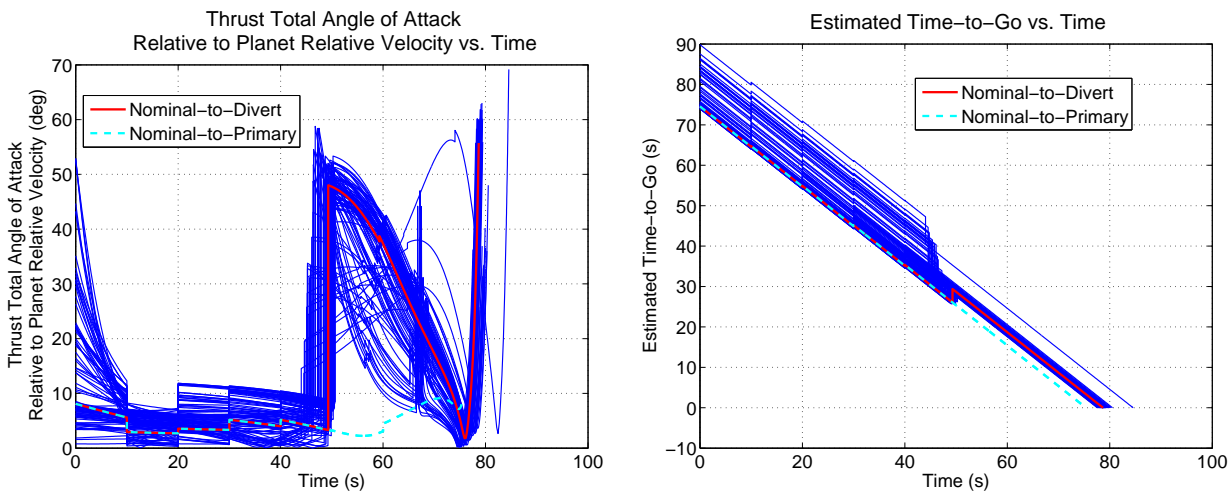

(o) Divert Target: Thrust Total Angle of Attack (p) Divert Target: Estimated Time-to-Go vs. Relative to Planet Relative Velocity vs. Time Time 